\title{
Polarized Solid State Target
}

\author{
Hartmut Dutz ${ }^{1, \star}$, Stefan Goertz ${ }^{1, \star \star}$, and Werner Meyer ${ }^{2, \star \star \star}$ \\ ${ }^{1}$ Physikalisches Institut, Universität Bonn, Nussallee 12, 53115 Bonn \\ ${ }^{2}$ Institut für Experimentalphysik - AG I, Ruhr-Universität Bochum, Universitätsstraße 150, 44780 Bochum
}

\begin{abstract}
The polarized solid state target is an indispensable experimental tool to study single and double polarization observables at low intensity particle beams like tagged photons. It was one of the major components of the Crystal-Barrel experiment at ELSA. Besides the operation of the 'CB frozen spin target' within the experimental program of the Crystal-Barrel collaboration both collaborative groups of the D1 project, the polarized target group of the Ruhr Universität Bochum and the Bonn polarized target group, have made significant developments in the field of polarized targets within the CRC16. The Bonn polarized target group has focused its work on the development of technically challenging polarized solid target systems towards the so called ' $4 \pi$ continuous mode polarized target' to operate them in combination with $4 \pi$-particle detection systems. In parallel, the Bochum group has developed various highly polarized deuterated target materials and high precision NMR-systems, in the meantime used for polarization experiments at CERN, JLAB and MAMI, too.
\end{abstract}

\section{Introduction}

Within the recent decades polarized solid targets as well as polarized particle beams evolved into indispensable tools in order to explore the spin structure of the proton and the neutron. Single and double polarization measurements were performed with a variety of different particles beams, among them not only experiments with polarized electrons and real photons. Protons, antiprotons and even polarized muons have been used so far at various laboratories [1]. (Double) polarization experiments at ELSA [2, 3] with tagged photon beam fluxes in the order of some megahertz have to rely on polarized solid targets with high target densities in order to provide sufficient luminosities of the particle reactions of interest. The developments of the polarized targets for particle physics experiments at ESLA within the CRC16 were twofold. On the one side we focussed on the enhancement of the figure of merit (FOM), which is defined as the product of the number $n_{t}$ of target particles per $\mathrm{cm}^{3}$, the squared target polarization $P_{t}^{2}$ and the squared ratio $f^{2}$ of the number of polarizable nucleons and the total number of nucleons in the target. Improvements in $P_{t}$ and $f$ are directly related to the search and preparation of suitable target materials and need a detailed understanding of the dynamic nuclear polarization (DNP), as given by the spin temperature theory (for an overview we refer to [1] and for deeper studies see [4]).

On the other hand we worked on a significant enhancement of the luminosity $L$ and angular acceptance $\Delta \Omega$ of the polarized target beyond the standard 'frozen spin technique' towards the so called 'large angular continuous mode target' or ' $4 \pi$ continuous mode polarized target'. The goal is to enhance the reaction counting rate $N$, which is defined as $N=L \cdot d \sigma / d \Omega \cdot \Delta \Omega$, and thus to improve considerably upcoming polarization experiments. The new scheme led to challenging and outstanding developments in cryogenics and superconducting magnet technology in the field of polarized solid state targets.

Besides the developments of new polarized target components and materials we operated the existing ' $\mathrm{CB}$ frozen spin target' (former 'GDH frozen spin target') as a major component of the Crystal Barrel experiment at ELSA for (double) polarization experiments.

Over the period of the CRC the goals and methods of the Bochum polarized target group focused on intense research on polarized target materials and their polarization behavior and the design and construction of more accurate as well as sensitive polarization measurement equipment. The broad spectrum of research covered the following topics:

- construction of a 'top-loading' ${ }^{4} \mathrm{He}$ refrigerator for target material research

- pulsed and CW NMR for polarization measurements $[5,6]$

- polyethylene and other oxygen-free hydrocarbons (alkanes) as polarized target materials

- radiation resistance of target materials with spinless spectator nuclei for high intense particle beam experiments

\footnotetext{
^e-mail: dutz@physik.uni-bonn.de

$\star \star$ e-mail: goertz@physik.uni-bonn.de

$\star \star \star$ e-mail: meyer@ep1.rub.de
} 
- conventional X-band and high field elctron paramagnetic resonance (EPR) measurements for systematic target material studies

Apart from the performance of the refrigerator and from the strength of the magnetic field, the achievable maximum polarization in a certain target material strongly depends on the efficiency of the DNP process. This efficiency is intimately related to the properties of the paramagnetic centers, from which the polarization is transferred to the nuclei. In particular the longitudinal Zeeman and the dipolar relaxation times as well as the strength of the electronic non-Zeeman interaction determine the minimum spin temperature and thus the maximum polarization of the nuclear spins. Therefore, to optimize the performance of a given target material, it is essential to characterize the paramagnetic system generated by the various doping methods as completely as possible. Meanwhile, besides a conventional X-band $(10 \mathrm{GHz})$ EPR spectrometer, which has been used since several years and which has produced a lot of very useful results (e.g. radical characterization and structure studies [7] or observation of spin temperature in ${ }^{6} \mathrm{LiD}$ [8]), also a V-band $(70 \mathrm{GHz})$ spectrometer is in use in the Bochum polarized target laboratory [9]. This self-built device is implemented in the ${ }^{4} \mathrm{He}$ polarization apparatus and enables EPR measurements in the DNP regime at $2.5 \mathrm{~T}$ and down to $1 \mathrm{~K}$. Especially for deuterated target materials very important results concerning the correlation between the properties of the paramagnetic species (i.e. EPR linewidth) and the maximum achievable deuteron polarization have been obtained at DNP relevant magnetic fields (see Fig. 1).

- Magnetic field dependence of the polarization in nitroxyl and trityl doped deuterated alcohol materials With respect to the projected ' $4 \pi$-continuous mode' polarized solid state target, it is of great importance to obtain an extensive knowledge about the polarization behavior within the moderate magnetic field region between $1.8 \mathrm{~T}$ and $2.5 \mathrm{~T}$. Since with internal polarizing field values beyond $2.5 \mathrm{~T}$ will probably not be accessible in the near future experiments with such targets will have to rely on a thorough optimization of the polarization conditions in order to achieve polarization values as high as required. Such an optimization means to check material parameters like radical concentration, EPR line-width and electron as well as nuclear relaxation times in dependence of the magnetic field with the goal to pin down an optimal set of parameters for each material. On the other hand the results of such measurements will help to specify the requirements for the construction of an internal polarizing magnet in terms of magnetic field values and magnetic field homogeneity. A new top-loading ${ }^{4} \mathrm{He}$-refrigerator capable to be operated up to 7.0 T has been successfully set into operation. Measurements of the electron relaxation time at $2.5 \mathrm{~T}$ have been performed in the very homogeneous magnetic field $\left(\Delta B / B<10^{-5}\right)$. For this a new pulsed nuclear magnetic resonance (NMR)-system has been built, which is able to measure relaxation times in the order of

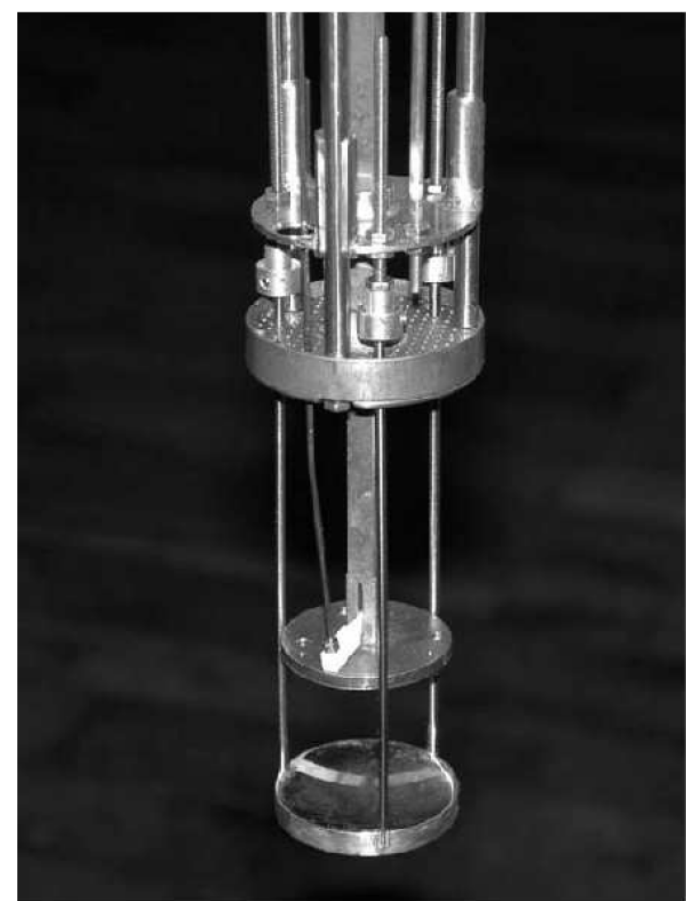

Figure 1: The Fabry-Perot like multimodal resonator of the high field EPR spectrometer (diameter of mirrors: $31 \mathrm{~mm}$ ). Because at $2.5 \mathrm{~T}$ the wavelength of the microwaves equals the dimensions of the solid target samples, a common Single Mode Cavity cannot be used.

milliseconds [10]. This is the first time that such measurements have been performed with samples used for particle physics experiments.

Furthermore a new continuous wave NMR-system was built, with which the degree of polarization of the polarized target materials can be detected with improved precision in the frequency range between $10 \mathrm{MHz}$ and $300 \mathrm{MHz}$ (corresponding magnetic field strengths between $1.8 \mathrm{~T}$ and $7.0 \mathrm{~T}$ for protons and deuterons, respectively). This development was necessary, as for the existing continuous wave NMR-system (Liverpool box) components are obsolete and other parts not available anymore on the electronic market (see Sec. 7.

A microwave system with a center frequency of $56 \mathrm{GHz}$ has been set up and is ready to work. Polarization values for protons at the corresponding field strength of $2.0 \mathrm{~T}$ and $1 \mathrm{~K}$ are expected to be in the order of some percent. To optimize the preparation of the samples for the particle physics experiments at temperatures $<80 \mathrm{mK}$ the ${ }^{3} \mathrm{He} /{ }^{4} \mathrm{He}$ dilution refrigerator in the Bochum laboratory has been modified and successfully operated for target material studies at low temperatures.

Since the Bochum group stressed the target material and NMR topics in polarized target research, the Bonn polarized target group put their emphasis more in the development of cryogenic components, small superconducting magnets as well as the operation of the 'CB frozen spin target': 
- design and construction of a new 'transverse holding-coil' and an 'internal superconducting polarizing magnet'

- construction of a new horizontal ${ }^{3} \mathrm{He} /{ }^{4} \mathrm{He}$ dilution refrigerator for the ' $4 \pi$-continuous mode' polarized solid state target

- set-up of a new irradiation facility for target materials at the $20 \mathrm{MeV}$ Linac of the Bonn accelerator facility and target material studies of irradiated polymeric materials.

In the following sections $2-7$ we will describe this work and recent results in more detail.

\section{2 'CB frozen spin target' at Crystal Barrel}

\subsection{Introduction}

The experimental program at the Crystal Barrel detector was focused on the measurement of single and double polarization observables off polarized nucleons using the existing GDH frozen spin target $[11,12]$, which with the start of the CRC16 has been labeled as 'CB frozen spin target'. Here we will briefly report on the setup and the performance of the 'CB frozen spin target' at the Crystal Barrel detector for polarization experiments at ELSA in the reporting period of CRC16.

\subsection{Setup and performance}

Upon completion of the GDH experiment at Mainz the target was moved back to Bonn and has been prepared for the experimental program at ELSA [13]. The main pumping unit was dismounted for revision and has been reinstalled to have a better access to the roots blowers to reduce the down time in case of a failure. In parallel the control unit was upgraded to guaranty a reliable operation of the system at ELSA. On top of that, the installation included a reconstruction of the mechanical support structure of the target, the railway system to move the polarizing magnet and the Crystal Barrel detector and the main vacuum system.

For the first run a butanol target material with low electron spin density $\left(1.4 \times 10^{19}\right.$ spins $\left./ \mathrm{ml}\right)$ was prepared to guaranty high relaxation times at moderate maximum proton polarizations. The relaxation time of the proton polarization during the first measurement at ELSA exceeded $500 \mathrm{~h}$ in a longitudinal 'holding field' of $0.64 \mathrm{~T}$. The nucleon polarization had to be refreshed every three days to minimize the beam time loss by the time consuming repolarizing process and movement of the detector components. The chosen frozen-spin cycle of about $72 \mathrm{~h}$ and a mean value of the target polarization at the beginning of the frozen spin mode of about $60 \%$ amounts to an average polarization of $57 \%$ during the data taking. After more than 8 years of successfull operation in the GDH experiment, the target has proven its known reliability. It is an ideal experimental tool to study spin dependent reactions in combination with a movable $4 \pi$-detection system.
To increase the reliability as well as the operational availability of the existing GDH frozen spin target, we replaced in the second funding period various hardware components of the control and vacuum-system of the refrigerator. Namely, the PLC- and HMI-systems were completely renewed and reprogrammed. Due to super-leaks in the front part of the target inserts, both units have been redesigned and reconstructed. We replaced the existing splitted NMRcoaxial cables in the inserts by only one. This led to a reduced heat load of the mixing chamber by longitudinal heat conduction and enabled us to improve the accuracy of the polarization measurement via the NMR-technique.

From 2008 to 2013 we run the frozen spin target for more than 15900 hours under cold operational conditions and could provide 7500 hours of longitudinally polarized protons up to a maximum polarization of $\pm 83 \%$ and deuterons up to $\pm 70 \%$ for single and double polarization experiments with the Crystal Barrel detector.

\subsection{Transverse 'holding coil'}

One of the major topics of the CRC TR16 was the measurement of the target asymmetry $\mathrm{T}$ and the double polarization observables $\mathrm{F}$ and $\mathrm{H}$ off the proton and neutron. These observables require a polarization direction perpendicular to the scattering plane of the pion nucleon system. To provide such a transverse polarization in the frozen spin target the existing 'longitudinal holding coil' had to be replaced by a new 'transverse holding magnet'. To ensure with the new set-up as long relaxation times as achieved with the 'longitudinal holding magnet', a field strength of at least $B_{H}=0.5 \mathrm{~T}$ is needed. And, as a crucial factor for a new magnet design, the maximum load of the current leads inside the dilution refrigerator is limited to $I_{H}=33 \mathrm{~A}$. The symmetry of the refrigerator, the available space in the target area as well as the cylindric form of the bobbin defines the 'transverse holding coil' as a race-track type superconducting magnet. In this design the magnet consists of two individual dipoles bend to a race-track each placed inside the bobbin. Additionally to the electrical parameters the magnet has to fulfill the requirements of a minimized mass distribution to ensure a good detection probability for the outgoing particles and the outer diameter of the magnet which is limited by the geometry of the refrigerator.

Taking these considerations and a superconducting wire with a diameter of $0.2 \mathrm{~mm}$ into account, the simulation of the magnet led to a solution for each coil consisting out of 510 windings within 7 layers. The combination of both coils result in a maximum field of the 'holding coil' of $B_{H}$ $=0.5 \mathrm{~T}$ at a nominal current of $I_{H}=30 \mathrm{~A}$. In contrast to comparable magnet designs, both coils were wound in a wet winding process. Thereby a cylindric shaped epoxy form was placed in between two race-track like guiding tools and used as a core for the winding of the wire. After hardening of the adhesive the core has been removed and both dipoles were placed and glued inside the bobbin. The fact that the bobbin of the new 'transverse holding coil' is identical in construction with the one of the 'longitudinal holding coil' allows us to change both magnets in short 
time (3 days). This gives us the opportunity to change the general settings and orientation of the polarization for a measurement of a new polarization observable. On the other hand the special design of the two dipoles inside of the bobbin leaves additional space for an optional solenoid wound on the outer shell of the bobbin. For the future one has with this double coil magnet the possibility to run a transverse and a longitudinal magnetic field to maintain the nucleon polarization in any possible angle to the scattering plane in the outgoing pion-nucleon frame by simply varying the currents in both magnets during the data taking.

In 2010 the new 'transverse holding coil' was used in the measurement of the target asymmetry $\mathrm{T}$ and the double polarization observable $\mathrm{H}$ at the Crystal Barrel detector. In more than $4300 \mathrm{~h}$ of cold operation of the frozen spin target it has proven its reliability. At the nominal holding field of $B_{H}=0.5$ Tesla, mean relaxation times in the order of $370 \mathrm{~h}$ have been achieved. The maximum proton polarization was of the order of $\pm 83 \%$. In Fig. 2 the polarization history for three typical double polarization runs in 2009, 2010 and 2011 for longitudinal, transverse, proton and deuteron polarization are shown respectively.

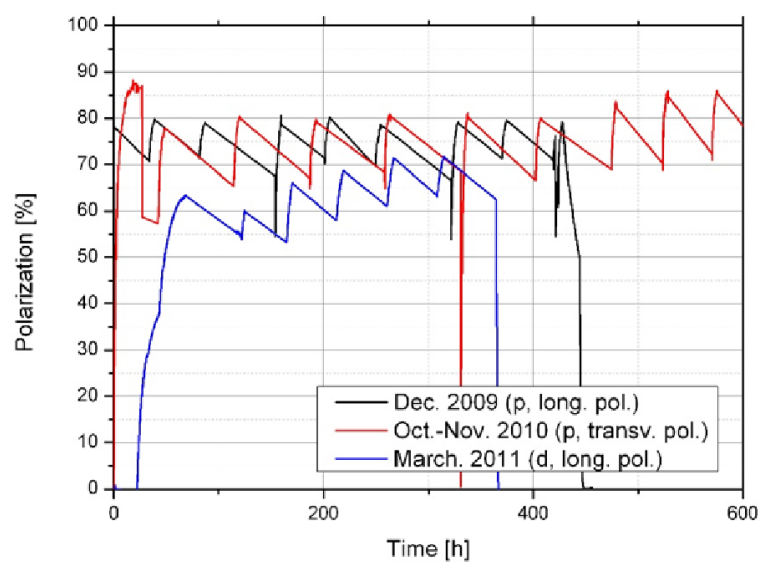

Figure 2: Polarization history plot for three typical double polarization runs in 2009, 2010 and 2011 for longitudinal and transverse, proton and deuteron polarization, respectively.

\section{A new horizontal ${ }^{3} \mathrm{He} /{ }^{4} \mathrm{He}$ dilution refrigerator for a $4 \pi$-continuous-mode polarized target}

\subsection{Introduction}

Besides the advantage of the good angular acceptance, the frozen spin target has in comparison with a 'continuous mode target' the disadvantage of a reduced beam time efficiency. It is caused by the polarization refresh cycles and the movement of the polarizing magnet as well as the detector components. In best cases a beam time efficiency of about $80 \%$ is attainable.

The second restriction from the 'figure of merit' point of view is the decay of the polarization during the data taking in all target materials. The overall polarization of an experiment using a frozen spin target is given by the relaxation times and limited to approximately $0.8 \cdot P_{\max }$. A reduction of the target temperature to increase the relaxation times is suppressed by the Kapitza-resistance in the target material at a given beam intensity. Commonly used intensities up to $10^{7}$ particles/sec are setting the effective target material temperature independently of the lowest refrigerator temperatures (e.g. $20 \mathrm{mK}$ ) to about $50 \mathrm{mK}$ to $60 \mathrm{mK}$. Beam intensities above $10^{8}$ particles/sec are unattainable in the 'frozen spin mode' operation.

To circumvent the disadvantages of the frozen spin technique we have combined the advantages with those of the 'continuous mode' operating target to a so called ' $4 \pi$ continuous mode' polarized target [14]. It is simply a 'frozen spin target' equipped with an internal superconducting polarizing magnet for permanent DNP. This new polarized target scheme, which can be described as a continuous mode operating target with a large angular acceptance, leads to an improvement in the figure of merit by a factor of 2 compared to the existing 'CB frozen spin target'. The special features of the target are

- large angular acceptance $\sim 4 \pi$ (homogeneous mass distribution)

- high average polarization during the data taking (continuous DNP at high polarizing field)

- high luminosities $L$ up to $10^{33} \mathrm{~cm}^{-2} \mathrm{sec}^{-1}$

- good beam time efficiency (no maintenance work for the moving of components nor repolarization),

- no moving system for the polarization process required (no detector size restriction, fixed target-detector setup)

Once the system will have been installed in the Crystal Barrel detector it will provide highly polarized nucleons with good access to the target region for scattering experiments with real and virtual photons.

Nevertheless, at this point it has to be mentioned that the ' $4 \pi$ continuous mode' polarized target in the first version only can provide longitudinal polarized nucleons for the scattering experiment. The symmetry and geometry of the horizontal refrigerator anticipate a solenoid as polarizing magnet enclosing the target material and the mixing chamber. In case transverse polarization is needed the ' $4 \pi$ continuous mode' polarized target has to be operated again in the classic 'frozen spin mode' whereby the transverse field orientation to maintain the nucleon polarization has to be provided by an internal 'transverse holding magnet'. The target material will be polarized via DNP in the internal longitudinal polarizing magnet and then rotated and maintained by the internal holding magnet. It seems to be possible to combine both coils to a hybrid coil, but this will be subject of future developments.

The main tasks towards the new target scheme in the CRC16 was the construction of a new horizontal ${ }^{3} \mathrm{He} /{ }^{4} \mathrm{He}$ dilution refrigerator and the development of a suitable 
small superconducting high field magnet as internal polarizing magnet implemented into the refrigerator.

Since the geometry of the new refrigerator is comparable with the existing one of the ' $\mathrm{CB}$ frozen spin target' the shape and the overall dimensions of the new internal polarizing magnet are the same as the actual internal holding coils. It has to fulfill the requirements of the high homogeneity of the external polarization magnets $\left(\Delta B / B \leq 10^{-4}\right)$ and the low mass distribution of the internal holding coils to ensure a good detection probability for the outgoing particles. A more detailed description of the development and the constrains of the internal polarizing magnet is given in section 5 .

\subsection{Design of the new horizontal ${ }^{3} \mathrm{He} /{ }^{4} \mathrm{He}$ dilution refrigerator}

The design, construction and setup of vertical as well as horizontal dilution refrigerators has a long and experienced tradition in the Bonn polarized target group and the precision workshops of the institute. Most of the components of the new refrigerator has been machined and built by staff members of our institute. The outer shape of the new refrigerator follows the design of the existing horizontal 'CB frozen spin target' dilution refrigerator. Various design aspects of our new cryostat have partly influenced the construction of the new horizontal dilution refrigerator of the 'Mainz Polarized Target' built by JINR Dubna. Differently from the existing type we focused on high cooling power $(200 \mathrm{~mW})$ in the temperature range at $250 \mathrm{mK}$ to $300 \mathrm{mK}$ to dynamically polarize an approximately $7 \mathrm{~cm}^{3}$ large target sample (DNP-mode). Nevertheless, to run the system optionally as a frozen spin target if transverse polarization is needed, we set the design base temperature to about $30 \mathrm{mK}$ to guaranty long relaxation times. As distinguished from the ' $\mathrm{CB}$ frozen spin target' dilution refrigerator we have added an additional ${ }^{4} \mathrm{He}$-evaporation refrigerator which encloses completely the main dilution refrigerator. This unit plays in first order the role as individual cooling sink for the internal superconducting magnet and its currend leads, in second order it is the thermal shielding for the complete system. The ${ }^{4} \mathrm{He}$-cryostat will be feed with liquid ${ }^{4} \mathrm{He}$ from the inner dilution refrigerator but nevertheless it can be operated independently from the inner cooling stages.

The beam line is the central part of the cryostat and has to fulfill the function of the internal isolation vacuum. The target material will be inserted into the refrigerator along the beam line by an insert that will have the same length as the one of the 'CB frozen spin target'. It will be vacuum tight against the mixing chamber by means of a cold indium seal in the still region. The precooling of the incoming ${ }^{3} \mathrm{He} /{ }^{4} \mathrm{He}$ mixture, liquefying at the thermal sink (1.2 K sink) will be comparable to the GDH-type. In contrast to the old refrigerator the first precooling stage is designed as a turbine like heat exchanger to improve the cooling power for the precooling and concurrently to reduce the ${ }^{4} \mathrm{He}$-consumption. The evaporator $(1 \mathrm{~K}$ pot $)$ is clasped around the still and has a volume of about 0.51 of liquid helium. The simulation of the precooling stages of the refrigerator is described in section 4 . It is the first computational fluid dynamics (CFD) simulation of a dilution refrigerator which takes all in- and backstreaming gases and fluids into account. The final heat exchanger and the still are in first order conditioned by the outer shape of the cryostat. From the outside, the system will look like a typical 'Roubeau - cryostat', the cylindric mixing chamber which is surrounded by the internal polarizing magnet is followed by the final heat exchanger. The low temperature heat exchanger was designed and delivered by the low temperature working group of the JINR Dubna. It is a 10 staged large surface sintered copper heat exchanger with an expected base temperature of about $30 \mathrm{mK}$. To the backward region the diameter opens conical to the pumping tee. The smooth transition from the larger diameter of the $70 \mathrm{~K}$ region to the low temperature part of the cryostat allows one to use high temperature superconducing (HTSC)-current leads for the internal polarizing magnet. An additional heat sink cooled by a separated ${ }^{4} \mathrm{He}$-circuit provide the required cooling power to cool the normal conducting current leads at the transition point to the HTSC down to $50 \mathrm{~K}$. Taking the parameter of a standard HTSC wire into account, a maximum current of $120 \mathrm{~A}$ can be accepted for an internal magnet. A cut-view of the new dilution refrigerator is shown in Fig. 3.

\subsection{Status}

We started with the design and first technical drawings of the refrigerator in 2006 and expected a construction time of 10 man years. After a successful start of the construction the history of this project is marked by various setbacks due to technical difficulties and staff changes in the precision workshops of the institute. The most crucial setback was caused by material faults in the thin wall stainless steel tubings of the already assembled precooling stages in 2013. This led to a series of vacuum leaks in the ${ }^{4} \mathrm{He}$ to ${ }^{3} \mathrm{He}$ heat exchangers although all components have been leak checked during the production process and later on during the assembling of the heat exchangers. We could reconstruct the leakages which were evoked by cavitation shrink holes (due to carbon cavities) in the tubings and the soft solder flux, as well as thermal stress during the TIGwelding. Approximately 3 to 4 month after the production process the shrink holes opened and cause the leakage. Unpleasantly nearly all leaks were unattainable for repair work, like additional soft or hard soldering. All this happened shortly before the first cool down of the cryostat. Consequently we decided to rebuild the complete precooling stage including a new central beam tube. All tubings have been replaced by new ones and each one was tested before the production process to build the new heat exchangers. As long as necessary, we have renewed soft solder connections and changed from TIG welding to laser welding. Since most of the components of the refrigerator as well as the custom made tooling for the heat exchangers, which had to be rebuild, were already built and tested we were able to complete the renewing and assembling of the 


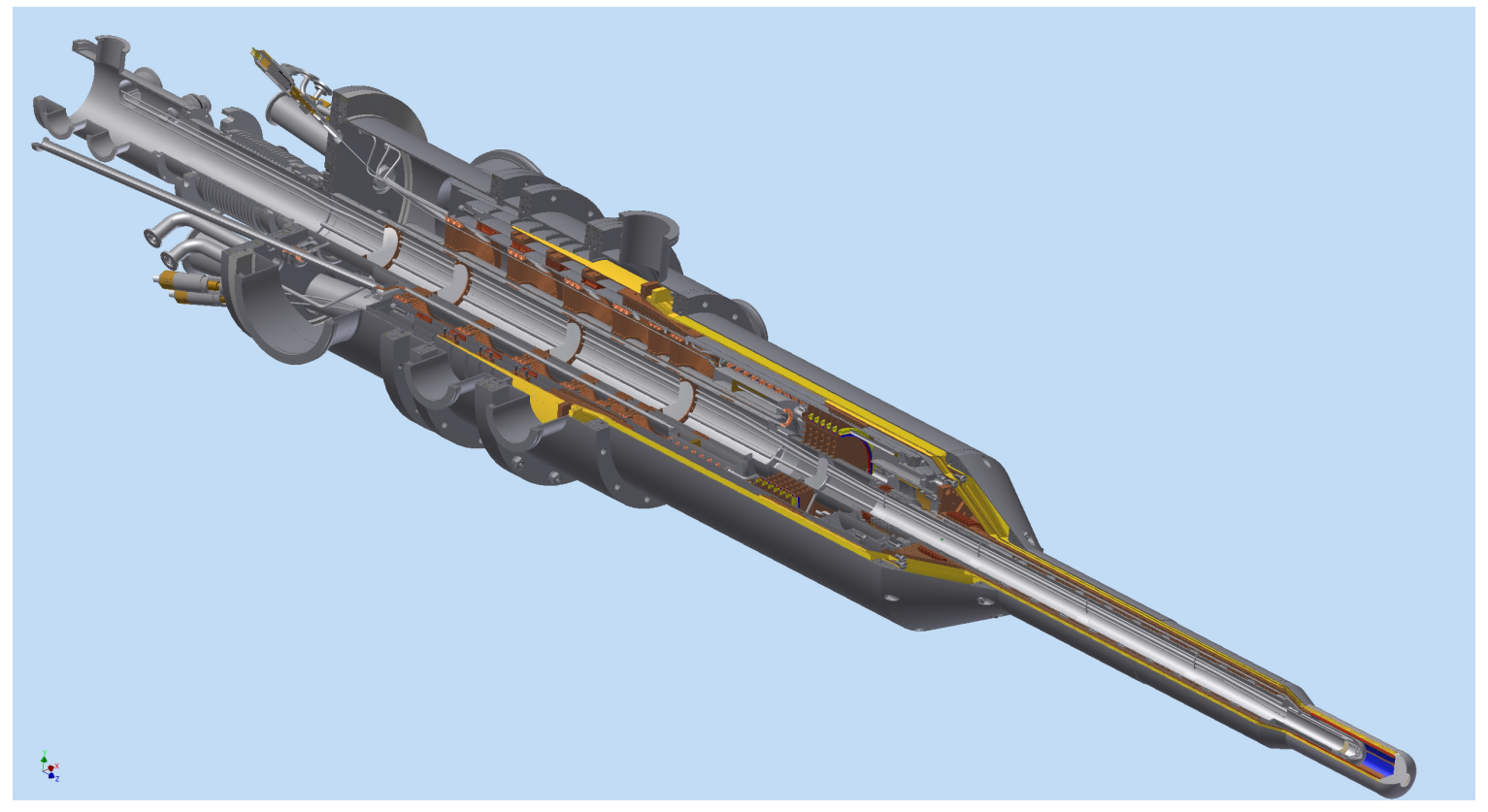

Figure 3: Cut view (technical drawing) of the new horizontal ${ }^{3} \mathrm{He} /{ }^{4} \mathrm{He}$ dilution refrigerator

precooling stages in fall 2015. Subsequently we started with the overall assembling of the precooling units, the dilution unit (still and low temperature heat exchanger), cooling shields and the outer vacuum envelope to complete the refrigerator. The first cool down of the completed system is now foreseen for September this year. We are still optimistic to get an innovative and reliable working dilution refrigerator for a new class of (double) polarization experiments within the scheme of the so called ' $4 \pi$ continuous mode' polarized target.

To cool down and cold test the refrigerator we have installed a complete test facility in our traget laboratory to avoid conflicts and overlaps with experimental program of the Crystal-Barrel experimental at ELSA. The test set-up is identical with the one at the CB area and it allows us to operate the new dilution refrigerator under typical run conditions at the beam area. As soon as we have reached the designed parameters and a stable operation at reasonable parameter setting, we will dynamically polarize the target material by means of the internal superconducting polarizing magnet. Once we have successfully demonstrated the feasibility of the new target scheme, we will move the target to the Crystal-Barrel experimental area for upcoming double polarization experiments.

\section{CFD Simulations of the new dilution refrigerator}

\subsection{Introduction}

Parallel to the design and construction of the new dilution refrigerator we started a program to study in more detail the fluid dynamics of a dilution refrigerator more general. Here the precooling stages of the refrigerator were of special interest. Since only rough calculations of the various precooling stages and types of heat exchangers can be found in the literature, we decided to perform a computational fluid dynamics (CFD) simulation of the single units and the combined precooling stage. The goal of the CFDsimulations is to estimate the characteristic flow parameter for every in and out going stream and to simulate the heat exchange between them for a better insight to the operational characteristics of this dilution refrigerator. Simulations for the different parts of the precooling stages were performed and will presented here in more detail [15].

\subsection{The precooling stages}

The precooling unit consists of two heat sinks and three heat exchangers which are shown in Fig. 4. One additional heat exchanger is located in the second heat sink. It is called evaporator and is working at a temperature of 1.2 K. HE1 and HE2 have to precool the incoming coolant (highly concentrated ${ }^{3} \mathrm{He}$ gas $(\approx 90 \%)$ and $10 \%{ }^{4} \mathrm{He}$ impurity at room temperature) by the usage of ${ }^{4} \mathrm{He}$ from the separator and the ${ }^{3} \mathrm{He}$ back stream coming from the still down to the separator temperature of $3.8 \mathrm{~K}$. Fig. 6 shows the necessary ${ }^{4} \mathrm{He}$ flow rate to cool the incoming ${ }^{3} \mathrm{He}$ gas down to this temperature. HE3 is supported by the liquid ${ }^{4} \mathrm{He}$ of the evaporator and liquefy the incoming coolant $\left({ }^{3} \mathrm{He}\right.$ gas). The heat exchanger in the evaporator should prevent the incoming stream from being cooled down to $1.2 \mathrm{~K}$ before it enters the dilution unit. Fig. 5 shows a flow scheme for the precooling unit.

The initial calculations for the heat stages of the precooling were done similar to [18-22] based on energy conservation for each stream. The main challenge for the calculation of heat exchangers used in horizontal dilution refrigerators is the determination of the heat exchange between 


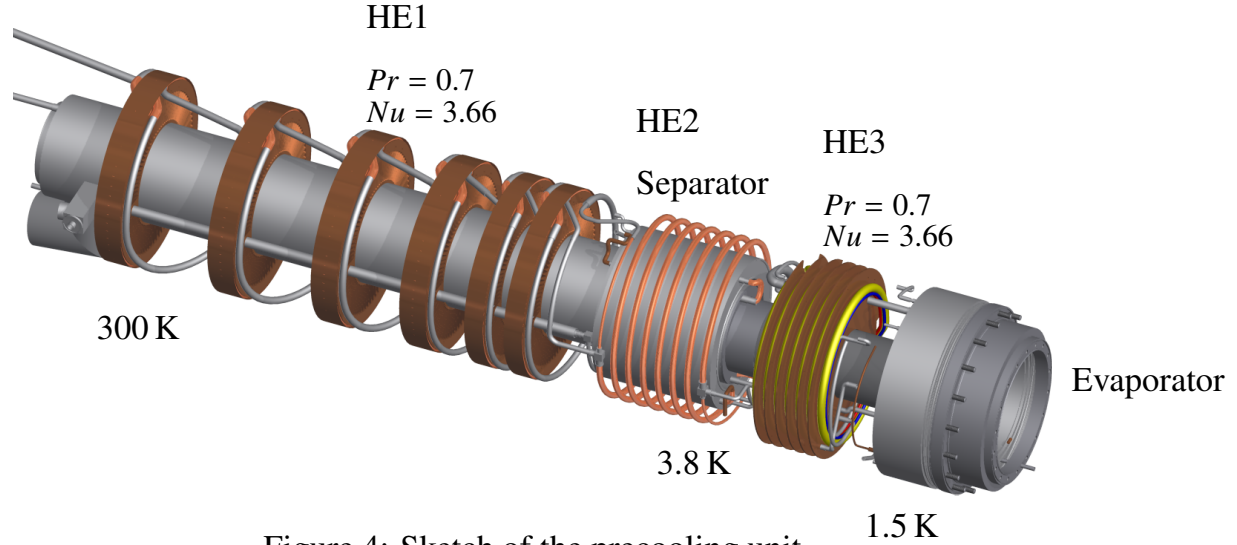

Figure 4: Sketch of the precooling unit

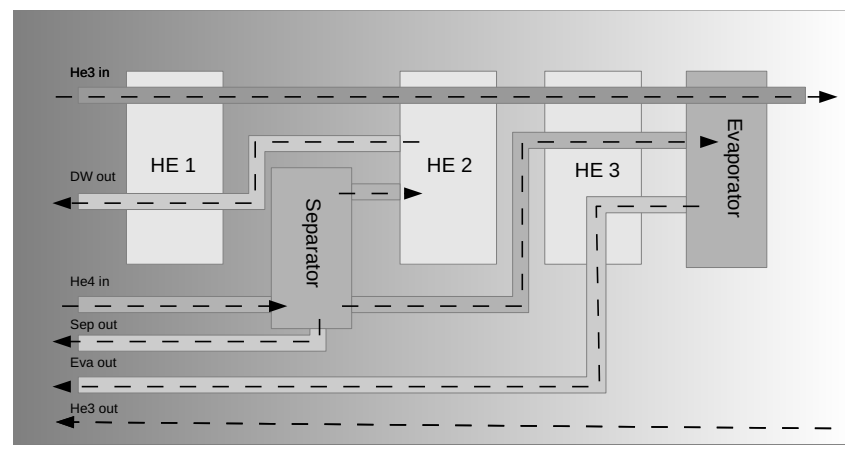

Figure 5: Scheme of the precooling unit and the different streams

the different streams through the solid

$$
\dot{Q}_{\text {solid }}\left(\Delta T_{\mathrm{m}}\right)=\alpha \cdot A \Delta T_{\mathrm{m}} \text { with } \alpha \propto N u(\operatorname{Re}, \operatorname{Pr}) \cdot \frac{\lambda}{L} .
$$

$\mathrm{Nu}$ is the Nusselt number, which depends on the Reynolds $(R e)$ and the Prandtl (Pr) number [20]. These three values are used to characterize the stream behavior. Equation 1 shows that the heat exchange depends on the characteristic flow parameter, the geometry (the surface $A$ and lenght $L$ of the heat exchanger as well as the thermal conductivity $\lambda$ of the material) and the temperature difference $\Delta T_{m}$. Unfortunately it can be a great challenge to determine this numbers. For the initial calculations the Prandlt and the REYNOLDS numbers for the backstreaming ${ }^{3} \mathrm{He}$ were estimated to $\mathrm{Pr}=0.7$ and $\mathrm{Nu}=3.66$. For the other streams the fluid flows through tubes and the calculations from [18] were used, for the calculation of $\operatorname{Re}$ and $\operatorname{Pr}$ see [19].

\subsection{CFD-Simulations}

CFD simulations were performed to estimate the influence of the backstreaming ${ }^{3} \mathrm{He}-{ }^{4} \mathrm{He}$ on the cooling power of the precooling stages. Almost all CFD simulations are based on $[16,17]$

$$
\frac{\partial}{\partial t}(\rho \phi)+\underbrace{\nabla \cdot(\rho \vec{u} \phi)}_{F_{\phi}}=D_{\phi}+Q_{\phi} .
$$

whereupon $\rho$ is the density, $\phi$ a fluid dynamic parameter (e.g. fluid velocity $\vec{u}$, pressure $p$ or temperature $T \ldots$ ), $F_{\phi}$ and $D_{\phi}$ are the convective and the diffusive flow and $Q_{\phi}$ represents all other distributions given by $\phi$. For a compressible fluid this leads to the

- continuity equation

$$
\frac{\partial}{\partial t}(\rho)+\nabla \cdot(\rho \vec{u})=0
$$

- momentum equation

$$
\frac{\partial}{\partial t}(\rho \vec{u})+\nabla \cdot(\rho \vec{u} \vec{u})=\nabla \cdot \tau-\nabla p+\rho \vec{g},
$$

where $\vec{g}$ represents body forces acting on the fluid contained in the control volume and $\tau$ the stress tensor which depends on viscous and pressure effects of the fluid.

- energy equation

Defining the specific enthalpy $h=u+p / \rho$ and introducing Fourier's law for heat transfer by conduction $q=-k \nabla T$, with the thermal diffusivity $k=\left(\mu c_{P}\right) / P r$, the dynamic viscosity $\mu$, the specific heat at constant pressure $c_{P}$ and the PRANDTL $(P r)$ number one can write the energy equation in the enthalpy form

$$
\frac{\partial}{\partial t}(\rho h)+\nabla \cdot(\rho \vec{u} h)=-\nabla \cdot q^{\prime \prime}+\frac{\partial p}{\partial t}+\nabla \cdot(\tau \cdot \vec{u}) .
$$

Depending on the specific case additional conditions like the equation of state or different specific material equations lead to a solvable problem. This simulations were performed with OpenFOAM [23]. The meshes for the calculation were created with the including mesh generating code called snappyHexMesh. There are two possible ways of simulation:

1. Single mesh simulations: a simulation of a single stream to get additional information about the characteristic flow parameters. This simulations are based on the solver rhoPorousSimpleFoam [23].

The advantage of this method is that higher mesh resolutions are possible and it is easier to determine the characteristic flow parameters like the PrandtL, 
the Nusselt and the Reynolds number. These values characterize the thermal coupling between the fluid and the solid in a system and if the flow is laminar or turbulent. The simulation can be performed for all streams until the flow parameters are known. In addition the characteristic flow parameters can be used to perform the calculations from section 4.2 again.

2. Multi mesh simulations: a simulation with a different submesh for the calculation of the heat exchange between the different fluid streams and solids. This simulations are based on the solver chtMultiRegionSimpleFoam [23].

By the usage of a multi mesh it is possible to set the boundary conditions for each stream in one simulation and run the solvers for each submesh representing a solid or a fluid. In this case the data necessary for the connecting surfaces of the submeshes can be replaced in each iteration and couple the solvers for each region. With this method the heat exchange between the different streams of the heat exchanger can be calculated in one simulation.

For both solvers we added the low temperature data from [25-27] to the thermo physical library implemented in these solvers. The viscosity of the helium gas was modulated with the SutherLAND equation [20]. The heat capacity $c_{\mathrm{p}}$ was assumed to be constant for the gaseous streams.

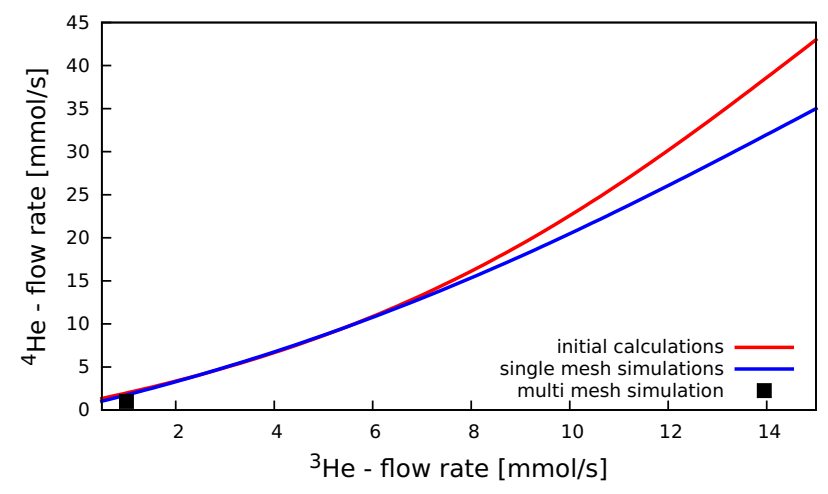

Figure 6: ${ }^{4} \mathrm{He}$ flow rate necessary to cool the incoming coolant to the temperature of the separator

\subsection{Single Stream Simulations}

In the beginning single stream simulations of the ${ }^{3} \mathrm{He}$ backstream were performed due to the lack of knowledge about the flow parameter of the backstream. Figure 7 shows the pressure and temperature gradient and the velocity profile as an example of this simulations. Here the backstream around heat exchanger 1,2 and the separator is shown. The beamline and the outer wall are expected to be adiabatic. The massflow is set to $1 \mathrm{mmol} \mathrm{s}^{-1}$ and the temperature is set to $3 \mathrm{~K}$ at the inlet. The heat transfer through the solids is given by the FourIER equation. For this simulation a constant mean temperature given by the calculations of section 4.2 was estimated for each cooling stage. These initial boundary conditions for the temperature are given in Fig. 7a.

The simulations were performed until a residual of $\leq 10^{-7}$ for all parameters was reached. Due to the heat exchange between the different streams these single mesh simulations have to be repeated until convergence for the complete system is reached. It is also possible to extract $R e$, $\mathrm{Pr}$ and $\mathrm{Nu}$ from these data. Afterwards the necessary ${ }^{4} \mathrm{He}$ flow-rate to precool the circulating coolant can be recalculated, this is displayed in Fig. 6 by the blue line.

\subsection{Simulation of a Heat Exchanger}

Multi region simulations were performed to calculate the heat transfer between the different streams and the important flow parameters of a heat exchanger in one simulation. In Fig. 8 and 9 the pressure distribution for the incoming and outgoing ${ }^{3} \mathrm{He}$ (coolant) is shown for HE2 as an example of this simulation. The temperature distribution for the different streams is given in Fig. 10 and 11. For the simulation shown in Fig. 8 to 11 a ${ }^{3} \mathrm{He}$ circulation rate of $1 \mathrm{mmol} \mathrm{s}^{-1}$ was estimated. The inlet temperatures for the ${ }^{3} \mathrm{He}$ backstream and the ${ }^{4} \mathrm{He}$ are set to $3.8 \mathrm{~K}$, the inlet temperature of the incoming ${ }^{3} \mathrm{He}$ is estimated to $5 \mathrm{~K}$. The simulations for all heat exchangers of the precooling stages with the flow rates for the different operation modes are ongoing. With this data a prediction for the necessary ${ }^{4} \mathrm{He}$ flow rate to precool the circulating ${ }^{3} \mathrm{He}$ under the influence of the ${ }^{3} \mathrm{He}$ backstream at a given circulating rate can be done. Fig. 6 shows the prediction from the multi region simulations for the flow parameter of successfully completed simulations at this time. Now it is possible to access the velocity profile, the temperature and the pressure gradient for all parts of a heat exchanger within one simulation. This information can also be used to optimize the performance of the different cooling stages by changing the geometry and by optimizing the flow impedances.

\subsection{Conclusion}

The polarized target group in Bonn developed a new horizontal continuous mode target for the $\mathrm{CB}$ experiment at ELSA. To increase the knowledge about the flow characteristics of a horizontal dilution refrigerator CFD simulations were set up. It is possible to use multi region simulations to calculate the performance of the different heat exchangers of a dilution refrigerator (see Sec. 4.5). This simulations are ongoing with all flow parameters, which can be expected for the new continuous mode dilution refrigerator for the CB-ELSA experiment. With this additional information we are able to optimize the concept of our dilution refrigerator which is under construction. After finishing the construction performance tests will be started and the experimental data will be compared with the simulations. 


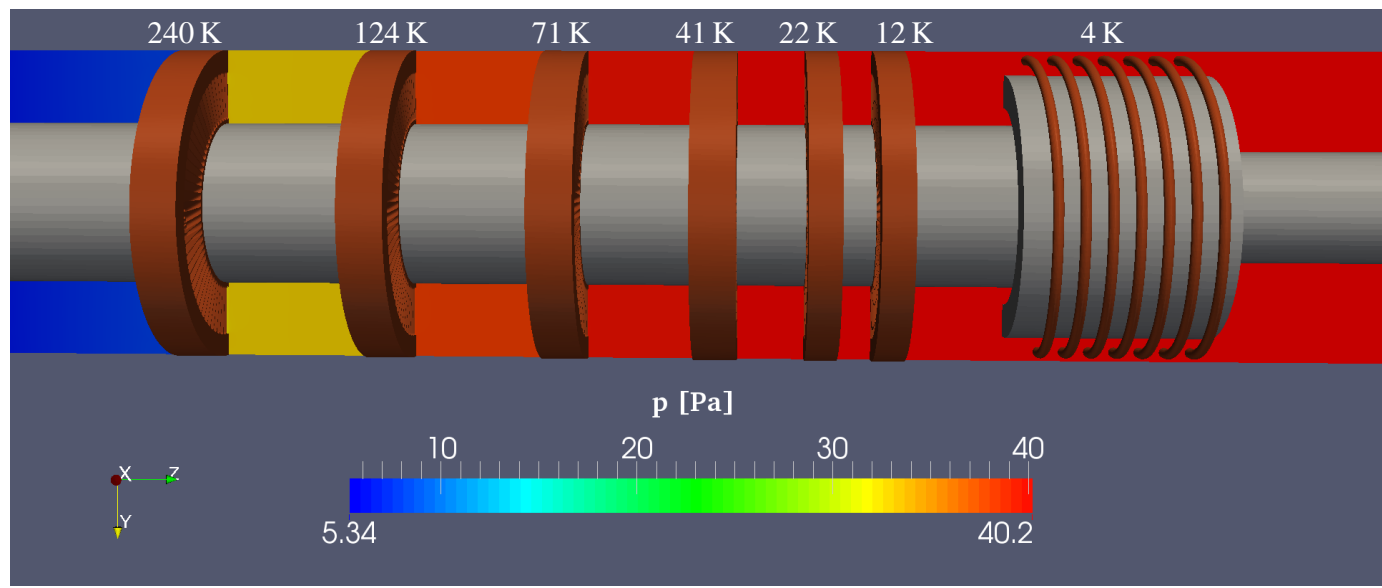

(a) Pressure distribution of the backstreaming ${ }^{3} \mathrm{He}$

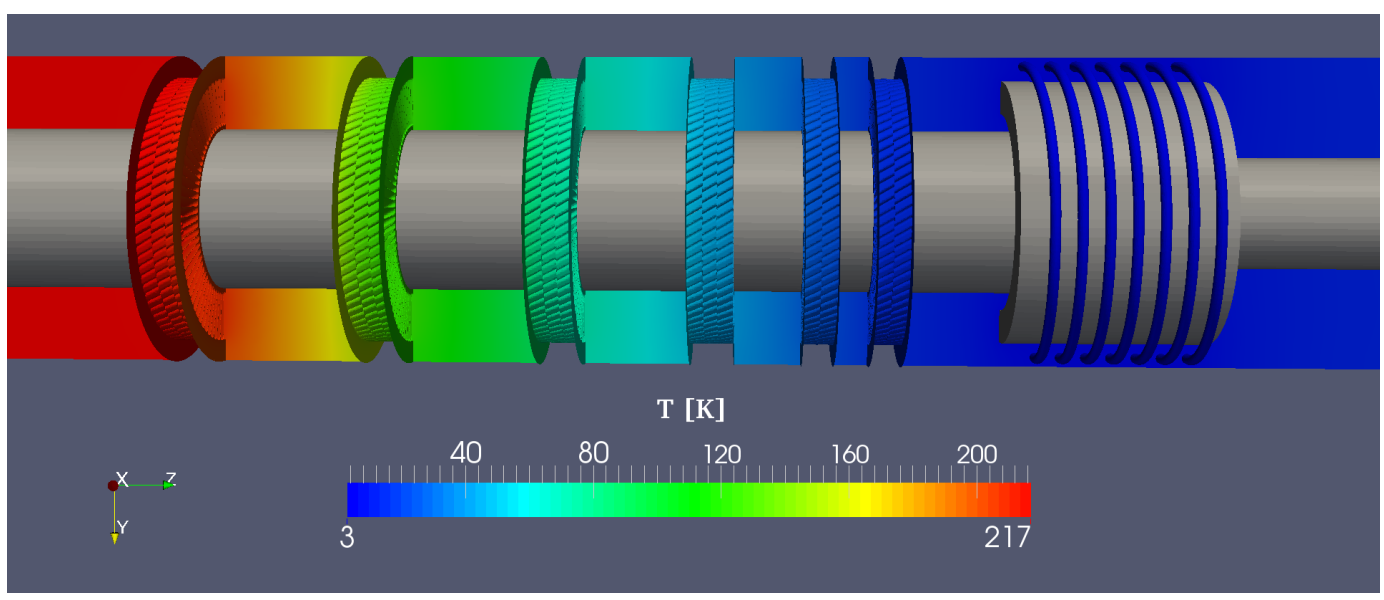

(b) Temperature distribution for the backstreaming ${ }^{3} \mathrm{He}$

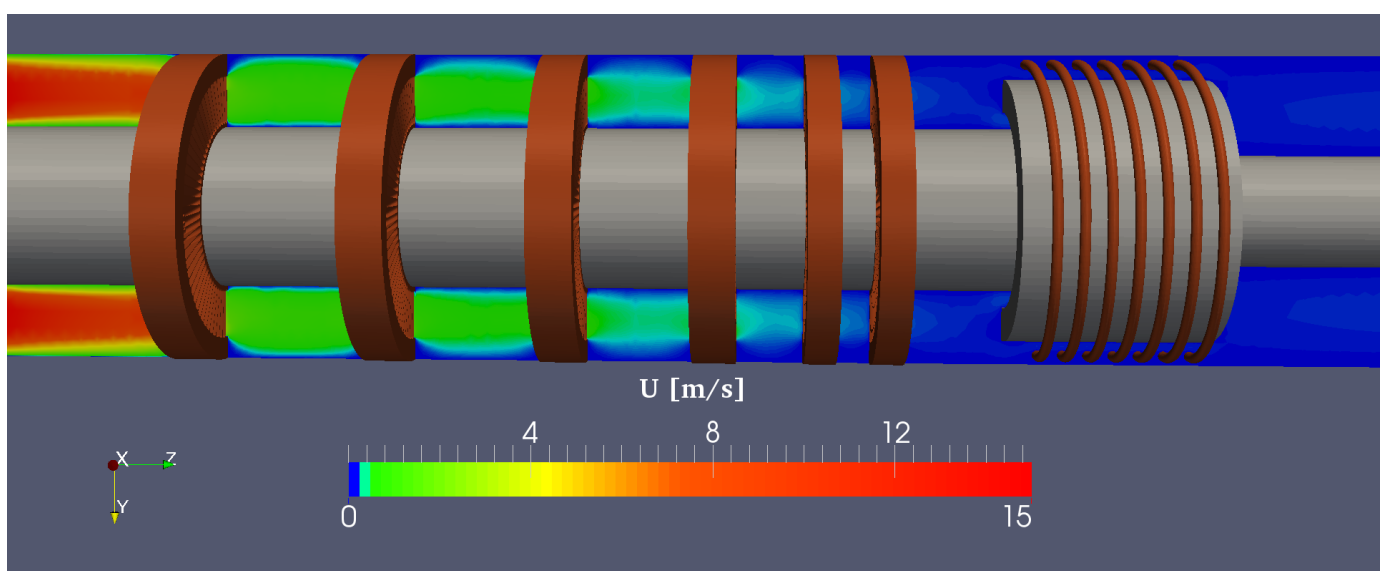

(c) Velocity profile of the backstreaming ${ }^{3} \mathrm{He}$

Figure 7: Simulation of the ${ }^{3} \mathrm{He}$ backstream for HE1 and HE2 at a mass flow rate of $1 \mathrm{mmol} \mathrm{s}{ }^{-1}$

\section{Development of a thin, internal superconducting polarization magnet for the Polarized Target}

\subsection{Introduction}

For improving the figure of merit of double-polarization experiments at CB-ELSA in Bonn the development of a novel Continuous Mode-Target has been proposed in 2004
[14]. One key element for this concept is a thin, high field polarization magnet, which is placed within the refrigerating structure. With such a magnet DNP could be used during the measurement of the experiment. A very first version of a 'thin high field internal magnet' (total thickness $1.3 \mathrm{~mm}$ [28]) has been wound for the existing 'CB frozen spin target' as holding coil and was in operation in nearly all double polarization measurements at ELSA.

Summarizing the first experience and tests with the inter- 


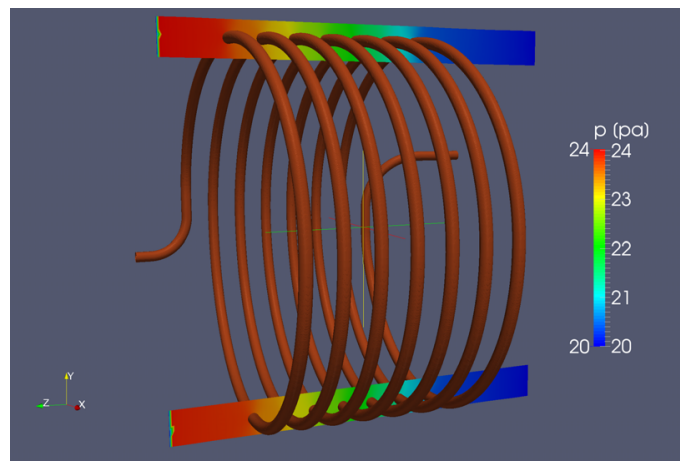

Figure 8: Pressure of the ${ }^{3} \mathrm{He}$ backstream in HE2

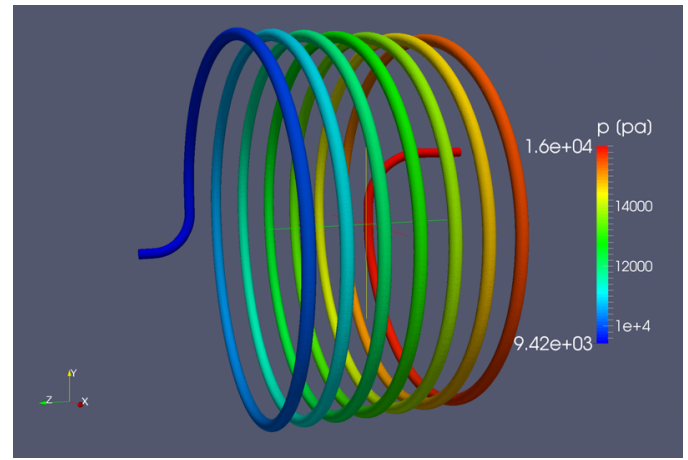

Figure 9: Pressure for the incoming ${ }^{3} \mathrm{He}$ stream (coolant) in HE2

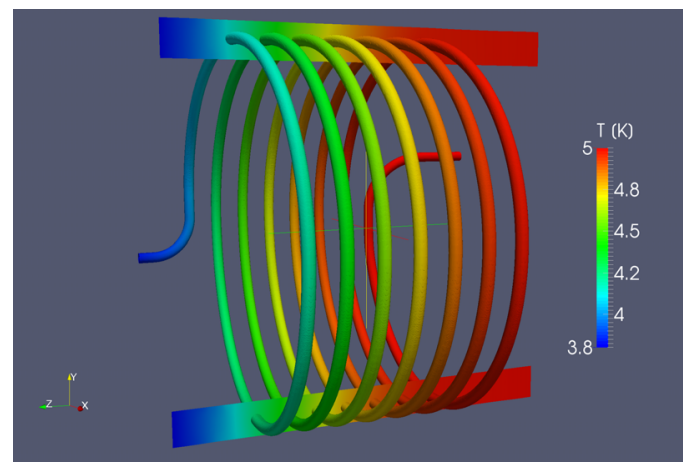

Figure 10: Temperature of the incoming and the outgoing ${ }^{3} \mathrm{He}$ stream in HE2

nal superconducting high field magnet, magnetic fields of $2 \mathrm{~T}$ are achievable in a thin coil with only 4 to 6 layers of superconducting wire at currents up to $100 \mathrm{~A}$. But with regard to the homogeneity of the magnet, the test measurements led to $\triangle B / B \approx 10^{-3}$, a more dedicated magnet simulation was required. The problems one has to deal with and which had to be solved within the simulation to improve the homogeneity are twofold. The specified minimized dimensions of the solenoid yield a ration between the magnetic volume and the target volume only of 0.05 which presuppose a calculation with respect to the accuracy of the positioning of the field correction elements as well as the positioning of each wire loop on the bobbin.

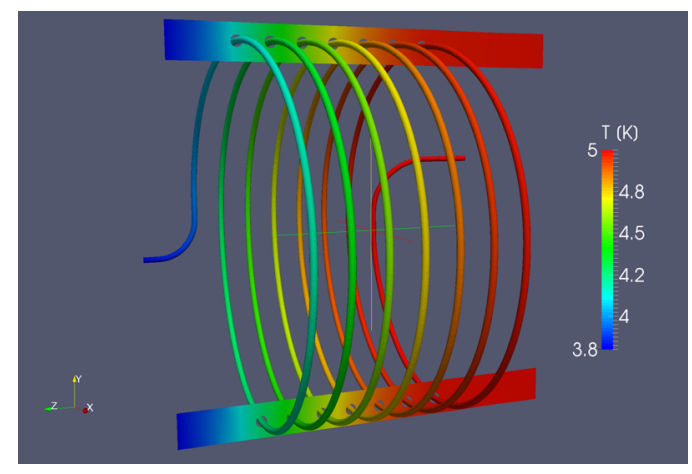

Figure 11: Temperature of the outgoing ${ }^{3} \mathrm{He}$ and ${ }^{4} \mathrm{He}$ stream in HE2

Finally one has to take the (limited) mechanical accuracy of the winding machine for the winding process of the superconducting wire into account.

The investigation of this problem in the framework of a diploma thesis has led to an 'inverse notched coil' corrected solenoid for the design of the magnet [29]. The simulated solenoid consist of 6 layers of superconducting wire $(\varnothing 254 \mu \mathrm{m})$ each with 590 windings. The corrector coils wound on the inner diameter of the solenoid ('inverse notched coil') (Fig. 12) consists of 16 windings within 2 layers. At a nominal current of $90 \mathrm{~A}$ a maximum field of $2.5 \mathrm{~T}$ should be achievable. The calculated homogeneity is of the order of $\Delta B / B \leq 5 \times 10^{-5}$.

The practical realization of the first design failed due to the limited mechanical accuracy of the winding process which was required to fulfill the high sensitivity of a stable solution of the homogeneity calculation. Therefore a new design concept with respect to the winding accuracy on the bases of the existing coil design was needed [30, 31].

\subsection{Boundary condition and design concept}

A technical drawing of the basic coil carrier can be seen in Fig. 12. The overall thickness of $2 \mathrm{~mm}$ should not be exceeded otherwise the detecting threshold will be raised by absorption of particles in the structure. For the DNP process a magnetic field of $2.5 \mathrm{~T}$ is needed. To fulfill both requirements a superconducting wire ${ }^{1}$ is used. The main field is created by a 6-layer solenoid with 586 windings per layer.

For polarizing deuterons the inhomogeneity (Eq. 8) of the field within the target area must be smaller than $\Delta B / B_{0}=$ $10^{-4}$. Because the field of a perfect solenoid is not sufficient $\left(\Delta B / B_{0}>10^{3}\right)$, two correction coils are added. The homogeneity is very sensitive to any misalignment of these coils. In contrast to the Notched Solenoid [32], where the correctors are wound on the outer shell of the main solenoid, they are placed in the inner shell of the solenoid. Two notches in the bobbin give maximum preciseness for positioning the coils (Fig. 12). Hence this concept is called the Inverse Notched Solenoid. Each correction coil has 2 layers with 9 windings each.

\footnotetext{
${ }^{1}$ multifilament $\mathrm{NbTi}, \varnothing=0.254 \mathrm{~mm}$ including insulation
} 


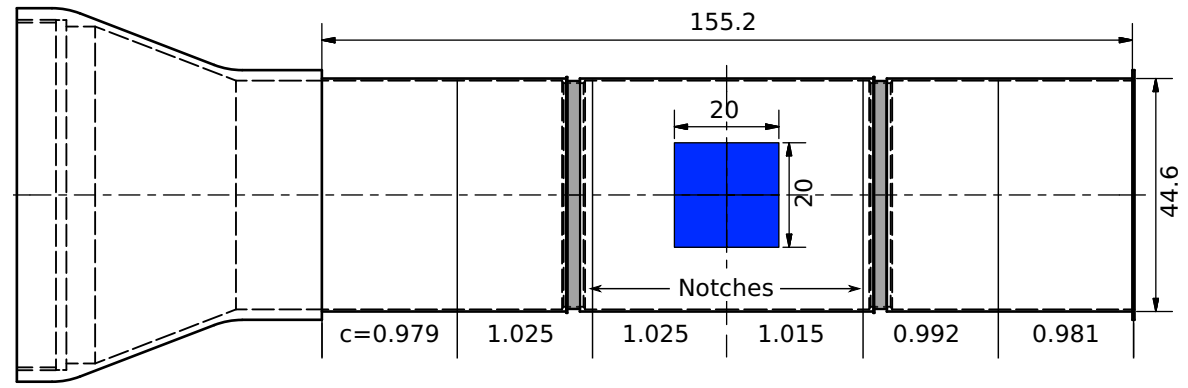

Figure 12: Sketch of the bobbin of an internal polarization magnet. The target area is located in the magnetic center. Within this area the magnetic field must fulfill the homogeneity criteria. The grey filled areas indicate the notches. The six values on the bottom side are the factors $c$ used for the density calculation (see Sec. 5.4.2). Length-units are in mm.

The whole magnet has an inductance of $160 \mathrm{mH}$ and a stored energy of $650 \mathrm{~J}$ at $2.5 \mathrm{~T}$. The wires are fixed to the carrier structure by a cryogenic epoxy glue in a wet wiring process.

\subsection{Calculation}

\subsubsection{Biot-Savart law}

For calculating the magnetic field of a solenoid and its correction coils the Biot-Savart-Law is used (eq. 6).

$$
\vec{B}\left(\overrightarrow{x_{0}}\right)=\frac{\mu_{0}}{4 \pi} I \int \frac{\left(\vec{\gamma}(t)-\overrightarrow{x_{0}}\right) \times \frac{\dot{\vec{\gamma}}(t)}{|\overrightarrow{\vec{\gamma}}(t)|}}{\left|\left(\vec{\gamma}(t)-\overrightarrow{x_{0}}\right)\right|^{3}} \mathrm{~d} t
$$

Figure 13 shows all necessary vectors for a certain path $\vec{\gamma}(t)$. For this design the path is given by a loop parametrization (Eq. 7) with $r$ as the radius of each loop and $d_{\text {eff }}$ (effective diameter, see Sec. 5.3.2) as the distance between two wires.

$$
\vec{\gamma}=\left(r \cos (t), r \sin (t), n \cdot d_{e f f}\right)
$$

The product of the counting variable $n$ and the effective diameter $d_{\text {eff }}$ gives the position of each loop. Due to the superposition principle the field of each loop may be added up to an overall field at a position $x_{0}$. To find the best position of the correction coils an algorithm loops over all possible positions and calculates the maximum field inhomogeneity (Eq. 8) within the target area. The central field $B_{0}=B(\overrightarrow{0})$ is used here as the reference value.

$$
\frac{\Delta B\left(\overrightarrow{x_{0}}\right)}{B_{0}}=\frac{B\left(\overrightarrow{x_{0}}\right)-B_{0}}{B_{0}}
$$

At the best position of the correction coils the field inhomogeneity can be calculated to $\Delta B / B_{0}<0.4 \times 10^{-4}$. Figure 14 shows the field map calculated for this case. The slight asymmetric structure comes from the fact that the magnetic center does not coincides with the geometric center. This is caused by the orthocyclic winding scheme (see fig.15) in combination of a constant loop number for each layer.

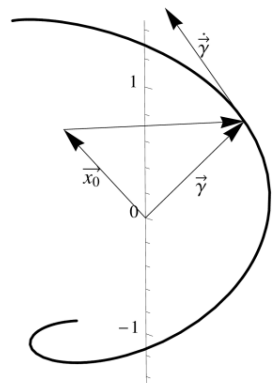

Figure 13: Electric current on a hectically path. The BiotSavart law is used for calculating the magnetic field $B$ at the position $\overrightarrow{x_{0}}$.

\subsubsection{Effective diameter}

The effective diameter $d_{\text {eff }}$ is defined as the distance between one wire and its neighbor. Because there is some space filled up with glue it does not coincide with the mechanical wire diameter $d$. As can be seen in Fig. 15 [33], in orthocyclic wiring the wire forms an equilateral triangle with $d_{\text {eff }}$ as edge length. The effective diameter mainly depends on the glue's viscosity, the tensile stress and (lateral) pressing forces on the wire. The latter is caused by the hand tool which applies the glue onto the surface and the efficiency of the wire guiding mechanism.

\subsection{First prototype}

\subsubsection{Estimation of the field homogeneity}

A first prototype of an internal polarization magnet was produced and tested in liquid helium at a temperature of $4.2 \mathrm{~K}$ and a cnetral field of $1.73 \mathrm{~T}$ at a nominal current of $65 \mathrm{~A}$ [30]. A hall probe could be moved along the central axis of the magnet for measuring the magnetic field $B$. The data are plotted in Fig. 16 with $B$ against the position $z$ on the magnet's central axis. As it can be seen there 


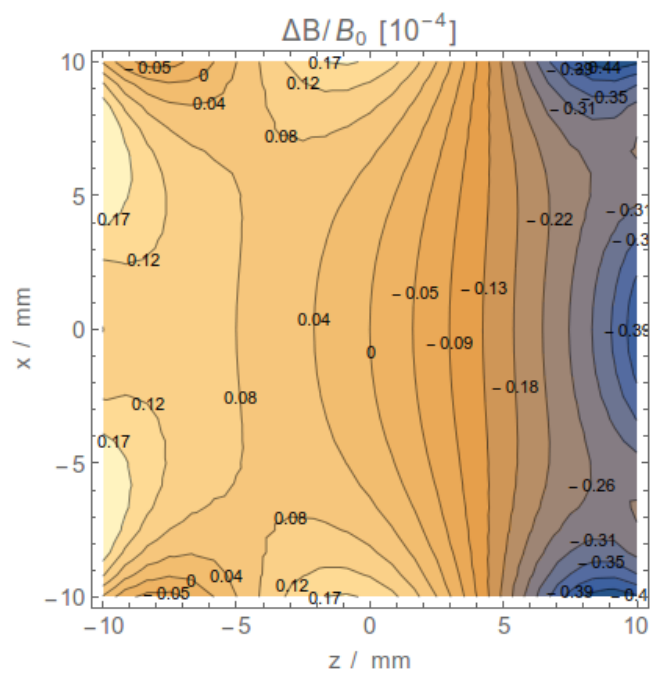

Figure 14: Field map of the target area of the 'Inverse Notched Solenoid'.

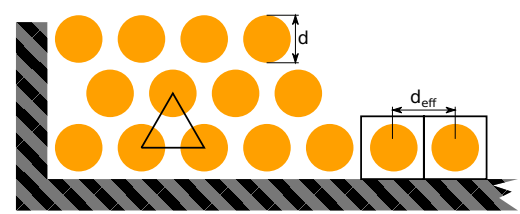

Figure 15: Schematic drawing of an orthocyclic wiring.

is a significant discrepancy between the measured and the calculated field. An asymmetric structure with two local maxima can be seen near the position of the correction coils. From this data it is clear, that the criterion of homogeneity has not been reached.

An explanation for this behavior can be found by a precise analysis of the wire position on the solenoid.

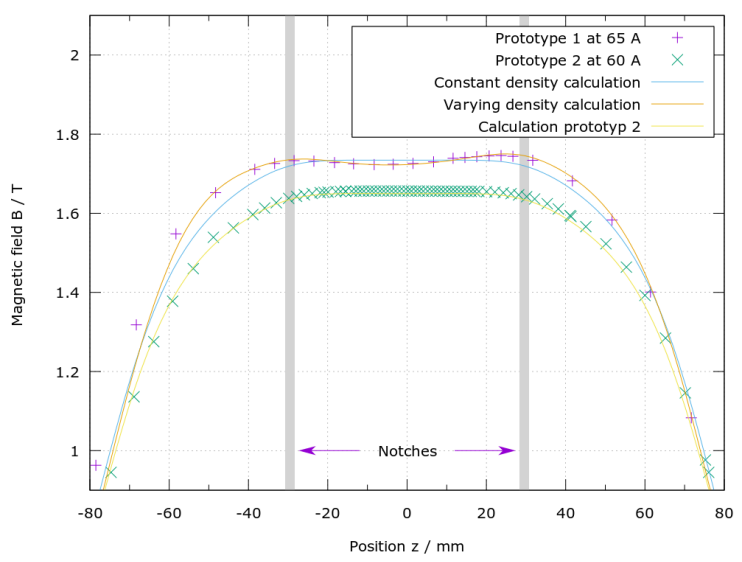

Figure 16: Comparison of the magnetic field measurement of the first and second prototype as well as the calculated (simulated) field for constant and distributed current density of prototype 1 . The grey filled rectangles illustrate the position and size of the two correction coils.

\subsubsection{Density calculation}

When calculating the magnetic field a homogeneous current density is assumed over the whole solenoid which presuppose a constant effective diameter. In practice the effective diameter can vary along the solenoid during the wiring process due to a non constant application of the glue to the wire. This lead to a local variation of the wire position compared to the calculated position and finally to a wire density variation which is equivalent to a local variation of the current density.

A simple model considering this effect is introduced in Fig. 12. The solenoid is split up into 6 parts, where the effective distance is modulated by a factor $c$. A factor $c<1$ means the effective diameter is reduced, the current density in this region is raised. As it can be seen in Fig. 16 this simple model can reproduce the measured asymmetric structure by varying the effective diameter only by about $2 \%$. The mispositioning of the wires and the non constant effective diameters could be verified by an optical microscopic scan of the magnet surface. The position of each wire has been determined afterwards with high precision by an image analyzing system.

As conclusion from these measurements one have to state, that the local variation of the wire has to be reduced otherwise the homogeneity will be diminished.

\subsection{Second protoype}

The variation of the effective diameter could be reduced to a value better than $1 \%$ by improving the wiring technique. Most important for a high quality winding process towards an orthocyclic wiring (Fig. 15 is a high precision wire guiding. In our case we used a custom made tool to guide the wire with a constant lateral force toward its neighboring loop. The feed speed of the guide was adjusted by a feedback control unit to the coiler speed, the viscosity of the used adhesive and the actual wired layer. All improvements of the new wiring technique lead to a second prototype of the "Inverse Noched Coil".

\subsubsection{Estimation of the field homogeneity}

\subsubsection{Field measurement}

The second prototype has been tested in liquid helium at $4.2 \mathrm{~K}$ with a current of $60 \mathrm{~A}$. The magnetic field distribution within the target area on the $\mathrm{z}$-axis has been measured using a standard hall probe at a maximum field of $B_{\max }=$ $1.65 \mathrm{~T}$. The data are plotted in Fig. 16. In contrast to the first prototype there is, as expected, a flat plateau within the target area. But due to the known limited resolution of the hall probe the homogeneity of the solenoid could be estimated not better than $\Delta B / B_{0} \leq 10^{-3}$.

For a further test the NMR-signals of a polyethylensample at $4.2 \mathrm{~K}$ and a center field of $B_{0}=1.599 \mathrm{~T}$ have been recorded. The cylindric sample with a diameter of $5 \mathrm{~mm}$ and a length of $22 \mathrm{~mm}$ could be moved along the the $\mathrm{z}$-axis. Figure 17 shows the normalized signals at different position. A frequency shift of each peak caused by 
the field inhomogeneity was compensated by adjusting the current resp. magnetic field to align each peak to the center frequency. As it can be seen in Fig. 17 this alignment could only be archived within a field band of about $0.35 \mathrm{mT}$ around the center frequency. The field deviation $\Delta B$ has been calculated from this current adjustment. The maximum field deviation close to the margin of the target area can be found at $\mathrm{z}=10.28 \mathrm{~mm}$ and is of the order of $0.20 \mathrm{mT}$. With regard to the uncertainty of the alignment the measurement lead to an estimated value for the homogeneity of about $\Delta B / B_{0}=3.5 \times 10^{-4}$.

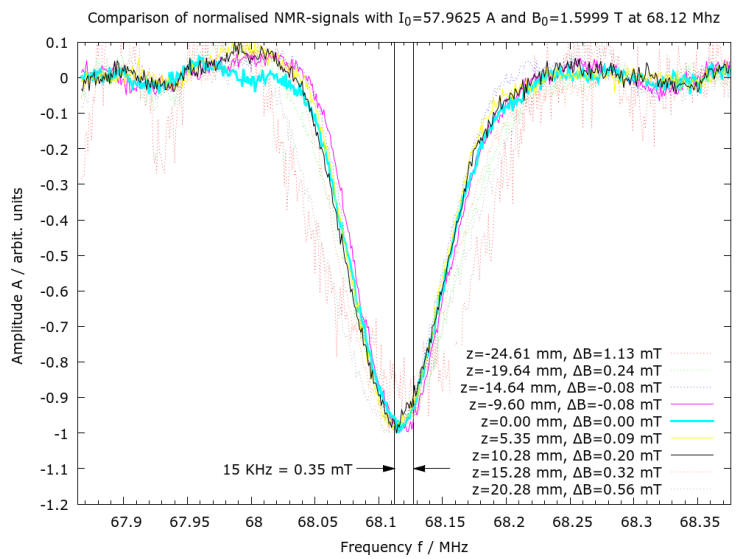

Figure 17: Normalized NMR-Signals of a polyethylene sample in thermal equilibrium in different positions within the magnet. All solid lines correspond to a position within the target area.

\subsection{Conclusion}

A thin, internal superconducting polarization magnet for the Polarized Target is actually in development. A magnetic field of $2.5 \mathrm{~T}$ with a field inhomogeneity better than $10^{-4}$ within the target area is necessary for the DNP process with deuterons. The basic concept - the Inverse Notched Solenoid - works with a solenoid for the main field and two correction coils which are added in the inner radius. This gives an accurate positioning of these correction coils.

Tests with the first prototype showed that the field inhomogeneity will definitely be influenced by a local variation of the wire position in the solenoid. To reduce the variation and to improve the accuracy of the wire positioning, a modified high precision wiring technique has been established. A second prototype has been build and passed first tests: The field measurement along the $\mathrm{z}$-axis shows the expected flat plateau within the target area. NMR-Signals of polyethylene at $4.2 \mathrm{~K}$ could be seen with this magnet and indicate a sufficient homogeneity.

For the next step the magnet will be installed in our ${ }^{4} \mathrm{He}-$ refrigerator. The maximum possible current will be determined at $1 \mathrm{~K}$. Additionally the magnet will be tested for dynamically polarizing a butanol-target.

Since the experimental program of the Crystal-Barrel ex- periment at ELSA has foreseen the measurement of double polarization observables on transversely polarized target nucleons, it is planed to design a combined coil providing a longitudinal high magnetic field for the dynamic polarization process and a weak transverse magnetic field to maintain the polarization in the so called 'holding' or 'frozen spin mode' of the polarized target. First preliminary design studies indicate the feasibility of this concept.

\section{Polarization and relaxation characteristics of irradiated polymeric materials at $1 \mathrm{~K}$ and at $2.5 \mathrm{~T}$}

\subsection{Introduction}

Polymers have been discussed as candidates for solid state target material for at least the last two decades. Unlike the commonly used alcohols e.g. butanol or inorganics e.g. ammonia, polymer targets have a major advantage in the fact that they can be formed to practically any desired geometry and can thus provide a filling factor close to 1 . In addition, the materials are solid at room temperature and handling is unproblematic. The defining point of a good solid state target material is a high polarization of the proton or deuteron components. The polarized target group at the Paul Scherrer Institute (PSI) showed dynamic nuclear polarization of protons in thin polyethylene (PE) foils and small tubes, chemically doped via diffusion of the highly stable free nitroxyl radical 2,2,6,6-tetramethyl-piperidine1-oxyl (TEMPO) to a spin density of $2 \times 10^{19} \mathrm{spins} / \mathrm{cm}^{3}$, to values of $70 \%$ in a $2.5 \mathrm{~T}$ magnetic field at a temperature below $0.3 \mathrm{~K}[34,35]$. Ideally one would like to achieve polarizations in polymer materials comparable to the classic materials of over $90 \%$. It has also been proposed that the crystallinity of polymers may in fact be a major factor in obtainable polarization values, as polarizations seem to be higher in amorphous materials than in crystalline materials at least with regards to chemically doped samples [36]. Kumada et al. [37] showed that for PE doped in the same manner as the PSI materials this was indeed the case. Their EPR studies showed that the chemical dopant TEMPO only diffused into the amorphous regions of the polymer. Whether or not the crystallinity affects the polarization if the radicals can indeed permeate the crystalline region, as is the case if the materials are irradiated, is still an open question [38].

\subsection{Polymer sample materials}

For the ongoing irradiation studies the focus was placed on two types of long chained polymer molecules: Polyethylene (PE) and polypropylene (PP). The density of both is comparable to that of butanol with a slightly higher dilution factor $f$ (see Sec. 1). PE is a long chain of carbon atoms, each with two associated hydrogen atom substituents $\left(\mathrm{CH}_{2}\right.$ monomer - see Fig. 18). For PP the situation is slightly different: The backbone of the molecule consists of carbon atoms, however next to every polyethylene monomer there is a carbon atom associated with a 
single hydrogen atom and a $\mathrm{CH}_{3}$ group $\left(\mathrm{CH}_{2}-\mathrm{CH}-\mathrm{CH}_{3}\right.$ monomer - see Fig. 19). The arrangement of the chains can be amorphous, the chains are random and unoriented, crystalline, the chains are folded and packed, or more commonly semi-crystalline in which crystalline structures are dispersed within the amorphous regions. Three differ-

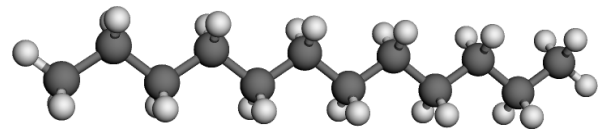

Figure 18: Polyethylene $\left(\mathrm{CH}_{2}\right)_{n}$

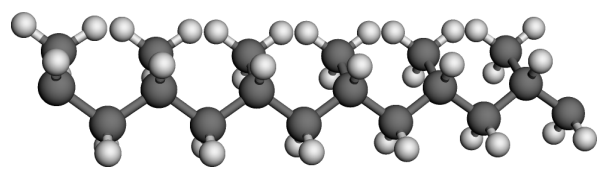

Figure 19: Polypropylene $\left(\mathrm{CH}_{2}-\mathrm{CH}-\mathrm{CH}_{3}\right)_{m}$

ent types of polyethylene were used.The samples consisted of 1) highly amorphous, low density PE (LDPE) with lots of branching, 2) a low branching, high density PE (HDPE) with a high amount of linearly arranged chains (crystalline) and 3) linear low density PE (LLDPE), a high branching material interspersed with small crystalline regions. Polypropylene is differentiated by its stereo regularity, the relative orientation of the $\mathrm{CH}_{2}$ and $\mathrm{CH}-\mathrm{CH}_{3}$ groups. Three isotactic materials of varying chain length were used for which all the substituents are located on the same side of the macromolecular backbone.

\subsection{Classification of samples after irradiation}

To achieve high polarization of nuclei, DNP is used to transfer spin polarization from electrons to nuclei. In order for this to be possible electron spins must be introduced into sample materials e.g. by the creation of persisting structural defects by irradiation. In theory hydrogen extraction and chain scissions can occur, however the former is by far more likely in polymer materials. After the irradiation the first step in the analysis of the materials is EPR spectroscopy. The comparison of the spectra of the irradiated material with spectra of a known spin density taken under the same experimental conditions gives access to the spin density of the irradiated material. This essentially tells us how many defects per unit weight were created in the irradiation process. Typical X-band spectra measured at a temperature of $77 \mathrm{~K}$ for PE and PP are given in figures 20 and 21. The EPR spectrum also gives insight into the type of paramagnetic centers that have been created. Understanding the underlying radical structure can help to optimize the temperature dependent irradiation process with regards to polarization. An alkyl-type radical (see Fig. 22) is created in irradiated PE and gives the sextet of the EPR spectrum: The electron is localized on a p-orbital of the single carbon atom and has a hyperfine interaction with the single $\alpha$ - and four surrounding $\beta$-protons [39],

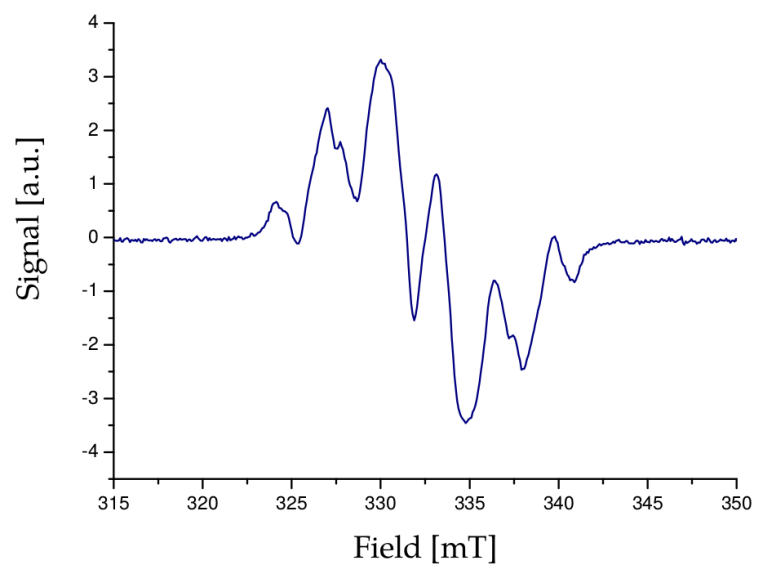

Figure 20: EPR of irradiated polyethylene.

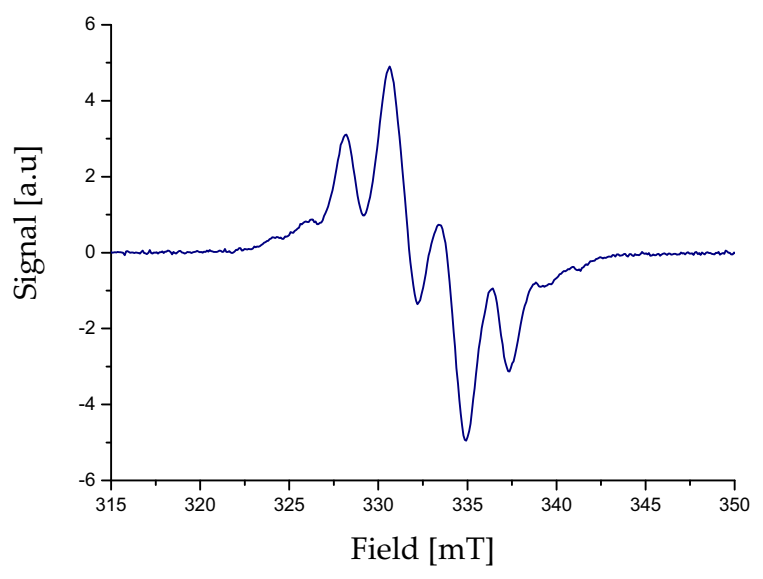

Figure 21: EPR of irradiated polypropylene.

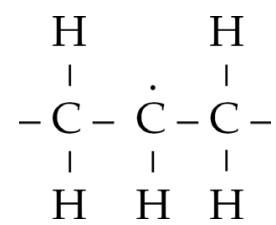

Figure 22: Primary radicals created by irradiation of PE.<smiles>CCC(C)C</smiles>

Figure 23: Primary radicals created by irradiation of PP.

[40]. On the other hand many structures have been proposed for the radicals resulting from the irradiation of PP, however a general consensus does not seem evident from the literature and major differences have been reported between spectra resulting from different types of irradiation e.g. $\mathrm{e}^{-}, \gamma$ and UV-sources. The main obstacle in determining the constituents of the spectra is the fact that irradiation 
of the material is thought to lead to a superposition of radical structures that are primarily obtained by the removal of hydrogen atoms. In polypropylene there are 3 sites from which a hydrogen atom can be removed, each of which would be expected to give an individual and distinct EPR spectrum. A hydrogen atom can be removed from the carbon backbone opposite of the methyl group or from the methylene bridge, as well as from the side-chain methyl itself. The most likely explanation is the dominance of the alkyl-type radical shown in Fig. 23 with additional structure resulting from the methyl group [41-44].

\subsection{Preliminary irradiations at the LINAC1}

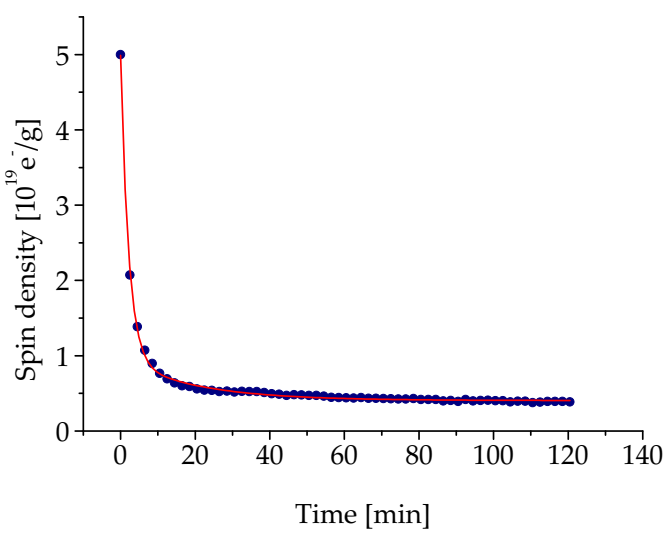

Figure 24: Decay of spin density in irradiated PP caused by heating of sample in nitrogen.

The electron irradiations in this study have so far been conducted in two consecutive data taking periods. Each of which will be described separately. The LINAC1 of the ELSA facility was used for the initial irradiation. A batch of PP pellets was irradiated in an argon cooled cryostat [45] and subsequently stored in liquid nitrogen. The initial spin density of the material was very close to 5 $\mathrm{x} 10^{19} \mathrm{e}^{-} / \mathrm{g}$ and in the order of magnitude in which one would typically expect DNP to be possible. As illustrated in Fig. 24 a subsequent heating of the samples in nitrogen at room temperature leads to a significant drop in the spin density. The initial rapid decay of the alkyl-type radical is followed by an almost stable region that lasts for some hours in which the alkyl-type radical is slowly converted into a allyl-type radical [46]. The conversion of the primary radical is evident from the slow increase of a sub-structure in the EPR spectrum, however the contribution of the latter to the spectrum on a whole is minimal and extremely difficult to extract due to the underlying noise. In this regard it is to be expected that any change in polarization characteristics of the material by heating are related to the primary radical. Two of the characteristics of interest for target materials are the nucleon relaxation time and the maximum obtainable proton polarization values. The change of these characteristics are shown in figures 25 and 26. Samples were analyzed at our standard ${ }^{4} \mathrm{He}$-refrigerator experimental conditions at a

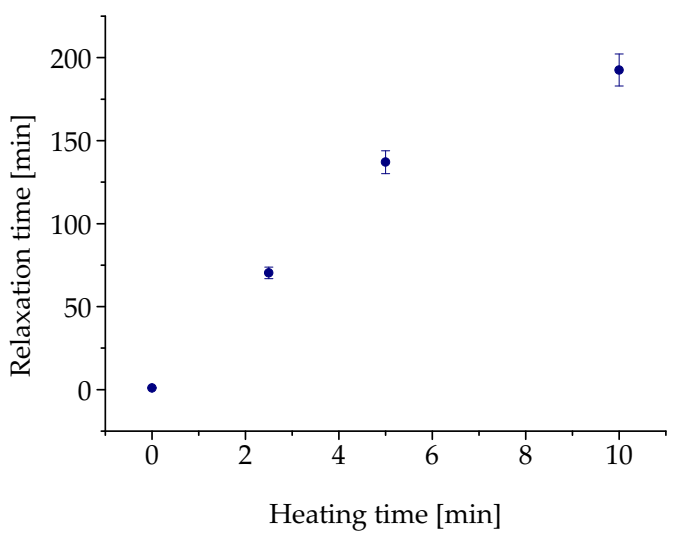

Figure 25: Relaxation times of irradiated PP after heat treatment.

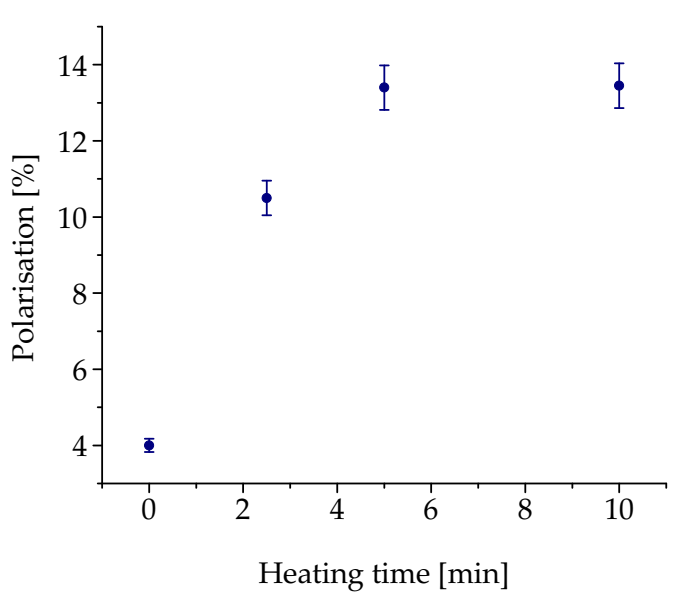

Figure 26: Polarization of irradiated PP after heat treatment

temperature of $1 \mathrm{~K}$ and a magnetic field of $2.5 \mathrm{~T}$. Samples were heated for $2.5,5$ and $10 \mathrm{~min}$. In these short periods of heating the change of the relaxation times is enormous. The non heated sample has a relaxation time in the region of a minute in comparison to approximately $70 \mathrm{~min}$ for the sample heated for only $2.5 \mathrm{~min}$. Additional heating increases the relaxation time further. The non heated sample gave maximum polarization values of $(4 \pm 0.2) \%$, with additional heating this was increased to $(13.4 \pm 0.6) \%$.

Unfortunately shortly after our introductory efforts the LINAC1 entered a prolonged period of maintenance and it was not possible to conduct any further irradiations of materials at this site: An alternative was needed.

\subsection{Present irradiations at the LINAC2}

In the past all irradiations of target materials in Bonn have taken place at the LINAC1 of the ELSA facility, however at present it is not possible to use this accelerator. Luckily, the second linear accelerator LINAC2 is well maintained and primarily provides electron beams for the Crystal Barrel and BGO groups. Modifications e.g. to the current beamline were needed, however it is now possible to extract the electron beam into the irradiation area. The end 
energy of LINAC2 is slightly higher than that of LINAC1 with $24 \mathrm{MeV}$ instead of $20 \mathrm{MeV}$, yet the beam intensity is 20x lower, meaning the irradiation times are significantly longer than in the previous irradiation of the polymeric materials $(10 \mathrm{~min})$, yet still not even close to the irradiation times of the non-organic materials such as $\mathrm{NH}_{3}$ or $\mathrm{LiD}(>10 \mathrm{~h})$. A typical irradiation time needed to obtain a nominal spin density of $10^{19} \mathrm{e}^{-} / \mathrm{g}$ is conveniently in the region of a few hours.

As described in the previous section, a heat treatment of irradiated samples enhances the maximum polarization values and increases the relaxation times. This positive effect should also be obtained by irradiating the materials above a material dependent specific temperature. In this way the "reorganization" of the electronic structure is obtained directly during the irradiation. In $20 \mathrm{~min}$ the change of the heated sample is more or less completed by the transition of the primary radical into a quasi-stable state. In the case of an irradiation the same (or a similar) radical can be created over a period of a few hours in a much more controlled way. To find the optimal temperature of irradiation a wide range cryostat was used [47],[48]. The cryostat uses a dual regulating system, implementing a slow cycled liquid nitrogen cooled heat exchanger coupled to a closed helium gas system with a fast regulating $1000 \mathrm{~W}$ heater. In this way it is possible to irradiate samples at temperatures from $90 \mathrm{~K}$ to room temperature with a stability of $\pm 1 \mathrm{~K}$. The lower beam current of the LINAC2, in comparison to the LINAC1, works in favour of the temperature stability. At present the work is very preliminary as we have only recently gained access to the facility and a broad outline of the programme will be given. Three different polyethylene samples, differing in density and linearity, and 3 different polypropylene samples, with varying chain length, have been irradiated simultaneously in the above mentioned cryostat. This ensures comparability of the samples: Any differences within a sample batch are thus due to differences in the materials. Irradiations were conducted at temperatures of $140 \mathrm{~K}, 180 \mathrm{~K}$ and $210 \mathrm{~K}$. The injected charge of approximately $3 \mathrm{mC}$ was sufficient to produce radical densities in the order of magnitude necessary for DNP.

As described in the previous section the first analysis of the materials was done with an EPR spectrometer and is summarised in Fig. 27. It was found that the spin densities of the different types of polyethylene are indistinguishable at the irradiated temperatures and thus the average was taken as a polyethylene batch. The same applies to the polypropylene samples. It is evident that the radical production mechanism in polypropylene is much more effective and the yield of radicals was around $40 \%$ higher. Over the analyzed temperature range there is a drop in the radical yield to higher temperatures. This is likely mostly due to the temperature dependent recombination process of the primary alkyl radical, as well as the conversion into a secondary radical to a far smaller proportion. As with the heated samples the latter can be seen the in EPR spectrum of the materials, however plays a subordinate role. The alkyl radical is still the dominant radical in all spec- tra. The analysis of the polarization values of the irradiated samples at a temperature of $1 \mathrm{~K}$ and a magnetic field of $2.5 \mathrm{~T}$ is currently in progress.

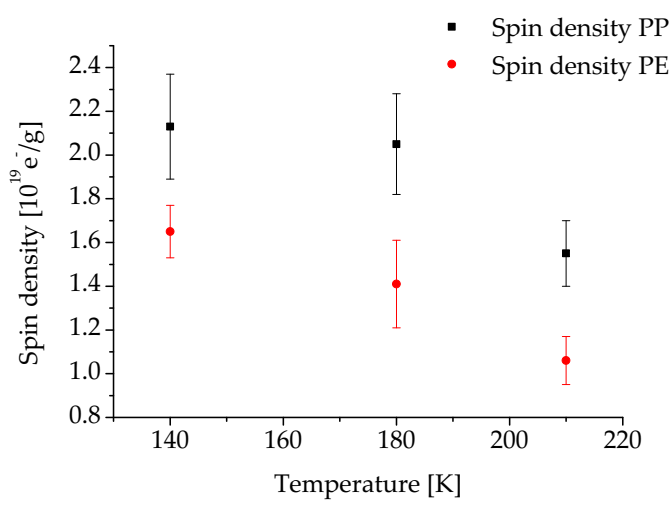

Figure 27: Spin density of PP and PE after irradiation at various temperatures.

\subsection{Conclusion}

As has been proposed in many papers, polymers can be used in particle physics experiments in which high polarization values are obtained using DNP. To what extent the polarization values are adequately high for practical use in such experiments remains to be seen, but previous results from chemically doped samples in the past and current results from the ongoing electron beam irradiations are very promising. The heating of the sample after irradiation has an effect on the relaxation and polarization characteristics of the samples. Significantly higher values of polarization are obtained after heating the sample, whilst relaxation rates become longer. The yield of radicals created as paramagnetic centers in PP by irradiation are in the region of $40 \%$ higher than in PE at the investigated irradiation temperatures. What influence the irradiation temperature has on the polarization characteristics is presently being studied: The change of the electronic structure initiated by heating should be obtained directly by irradiation at higher temperatures. Polarization measurements at very low temperatures are planned for the near future.

\section{Development of a new Q-meter module}

\subsection{Introduction}

In the research field of polarized target physics the Qmeter is a well established technique to determine the polarization of solid target materials [49]. One of the most common Q-meter modules is represented by the so called Liverpool-NMR [53], which was originally developed for the EMC experiment in the 1970s. However, since the module was built nearly 40 years ago, many components are no longer available. Thus the repair of existing modules and reproduction of new modules is impossible. Starting from that point, a new Q-meter module was developed and investigated for the use in polarized target experiments 
[50].

For the operation and evaluation of measurements in particle physics experiments with polarized solid targets a precise knowledge of the sample polarization $P_{t}$ is essential. This can already be seen by the so-called figure of merit

$$
\text { FOM } \sim f^{2} P_{t}^{2} \sim \frac{1}{t_{\text {meas }}}
$$

of a polarized solid target, which characterizes the quality of the used target material with a dilution factor $f^{2}$ and is anti-proportional to the required measurement time $t_{\text {meas }}$ for a given precision of these measurements.

In general, for the detection of target polarization the method of nuclear magnetic resonance is used. By means of a resonant circuit whose inductance is located in proximity to the sample, an alternating field is generated, which is oriented orthogonally to the external magnetic field $B_{0}$. If the frequency of the alternating field matches the Larmor frequency $\omega_{L}$ of the investigated spin system with the gyromagnetic factor $\gamma$,

$$
\omega=\omega_{L}=\gamma B_{0}
$$

transitions between the Zeeman levels are induced. However, this interaction only occurs for a narrow frequency range in the region of the Larmor frequency of the nuclei. Therefore, this effect is also called resonance absorption. Macroscopically, this results in a change of the sample magnetization, which is caused by the different occupied Zeeman levels of the sample.

Based on this change in magnetization, the NMR spectrum of the investigated sample can be determined. The area $A$ under the NMR line is proportional to the target polarization

$$
P_{t}=k \cdot A
$$

with the so called enhancement factor ${ }^{3} k$.

\subsection{NMR methods}

In principle, there are two different methods for the detection of the NMR spectra of a sample. For the socalled pulsed NMR method, which is the current standard in many scientific fields, the sample magnetization is deflected from its original position parallel to the external field $B_{0}$ by a short and powerful RF pulse. The subsequent relaxation of the magnetization back to equilibrium can be described by means of the Bloch equations [51, 52], leading to a voltage induction into the NMR coil. This timeresolved voltage signal is commonly known as free induction decay signal FID and can be converted by a Fourier transformation into the desired NMR spectrum. However, for the exact determination of polarization by this method there arise different problems, which are attributable to a

\footnotetext{
${ }^{2}$ The ratio of polarizable nuclei to the total number of nuclei in the target material

${ }^{3}$ This factor is usually determined by a calibration in thermal equilibrium, because the corresponding polarization $P_{t, T E}$ can be calculated for a given magnetic field and a given temperature, so that a simple assignment $k=P_{t, T E} / A_{T E}$ can be made. This method is also called TEcalibration
}

non-uniform signal excitation and a signal loss at the beginning of the FID detection.

Therefore, the well established continuous wave NMR technique is still the method of choice for the accurate detection of the undistorted NMR spectra and so for the measurement of the target polarization. Usually, this method is preformed at constant external field ${ }^{4} B_{0}$, while the frequency of the applied radio-frequency alternating field is swept over the resonance. Depending on the number and direction of the excited transitions between the Zeeman levels of the spin system, the power consumption or output of the resonant circuit changes. Since also the quality of the resonant circuit is affected, this set-up is also called Q-meter. The change of the quality of the resonant circuit can be detected as a variation of the radio-frequency power reflected from the oscillating circuit.

However, by this means there are two difficulties. On the one hand, radio-frequency signals are difficult to handle, on the other hand, the signal to detect consists of two signal parts and is complex. Only the area below the imaginary part of the signal, the so-called absorptive signal component, is proportional to the target polarization $P_{t}$. To determine the amplitude of the radio-frequency signal one uses a diode detector followed by a low-pass filter which converts the RF signal to a DC signal proportional to the $\mathrm{RF}$ amplitude. For the detection of the absorptive signal component, a phasesensitive detector is used, which is in phase with the exiting signal.

\subsection{Developement of a new cw NMR module}

Therefore, the requirement for a newly developed $\mathrm{cw}$ NMR module for polarization detection of solid targets was given, which should serve as a substitute for the Liverpool module. However, possible improvements should not be disregarded. To enable maximum compatibility with existing systems, the new development [54] is based in many ways on the construction of the Liverpool module. Specifically, these are the following:

- exciting RF signal via external generator

- two demodulation channels

- diode channel for receiving the magnitude spectrum and to tune the NMR resonant circuit

- phase-sensitive detector for the detection of the absorption spectrum

- layout of the connector for power supply and signal transmission

- geometric dimensions of the module

The main differences between the modules can be attributed to various improvements and the necessary replacement of no longer available components:

- outsourcing of the resonant circuit components - ensures greater flexibility in experimental set-up of the measuring circuit (e.g. $\lambda / 2$-cable)

\footnotetext{
${ }^{4}$ For the operation of a polarized target, this results in the advantage that the necessary dynamic nuclear polarization frequency $\omega_{D N P}$ is kept constant
} 
- the capacitive elements of the resonant circuit have been replaced by a varicap diode - this allows electronic tuning of the resonant circuit and an access to a measurement based on synchronous resonance tuning (see [55])

- except the resonant circuit components, all components are on one circuit board with a system-wide impedance of $50 \mathrm{ohms}$ - this allows a simple construction and repair of the modules, also possible signal reflections should be minimized

- replacement of the radio-frequency amplifier by common models from Mini Circuits - good signal-to-noise ratio and price-performance ratio

- exchange of rectifier diodes - granted a higher linearity of this channel (see below)

To shield the components against internal and external noise, the boards were placed in specially designed housings of solid brass, which were adjusted to each other.

\subsection{Test measurements}

For the warranty of functionality of the newly developed cw NMR module various test measurements were carried out. The most important ones are represented here briefly.

\subsubsection{Linearity}

A basic requirement for the exact detection of the target polarization over the area units $A$ under the NMR-line is only possible, if the amplification of the signals in the relevant performance range ${ }^{5}$ is constant. Therefore, measurements at different frequencies ${ }^{6}$ were performed to test the linearity of the two channels in this region. The NMR resonant circuit was replaced by a variable attenuator, so that different input power levels $(-62 \mathrm{dBm}$ to $-31 \mathrm{dBm})$ could be simulated.

Considering the resulting voltage profiles of the matched BRM channel in Fig. 28, a high degree of linearity can be seen in the work area for the different frequencies. The varying slopes between these traces at the given frequencies are due to different gains. This fact can be attributed to the frequency dependencies of the used components and is not important for the detection of the polarization at a fixed frequency.

Checking the linearity of the diode-channel based on BAT85-diodes, also used in the Liverpool module, there was a high deviation in a linear amplification at 212.6MHz. This effect could ultimately be attributed only to the diodes used. For this reason, various diodes were tested with respect to their linearity in the work region. It was found that the diode type SMS7630 which, according to data, is especially designed for mixers, shows the most promising behavior. Also measurements in the other relevant frequencies demonstrated the advantages of this type of diode, so that the linearity of this channel could be increased significantly.

\footnotetext{
${ }^{5}$ The input power of the NMR module during a polarization measurement is, according to experience, at about $-41 \mathrm{dBm}$.

${ }^{6} \mathrm{Larmor}$ frequency of deuterons and protons at $2.5 \mathrm{~T}$ and $5 \mathrm{~T}$.
}

\subsubsection{Detection of real spectra}

In addition to linearity, the possibility for the exact detection of the TE polarization plays an important role. For this reason, the TE signals of protons and deuterons of different samples were measured. As can be seen in Fig. 29 , even smallest signals become clearly apparent from the predominantly white noise of the circuit, so that an exact determination of area units in the thermal equilibrium is possible.

A particular challenge for the polarization detection is represented by the TE signals of quadrupole broadened deuterons, since these spectra are split over a wide frequency range and the corresponding signal amplitudes are small. Nevertheless, as illustrated by fig. 30, also these spectra can be well detected with the new module, so that a polarization determination based on the TE method is possible.

By the characteristic waveform, which is for example described in [56], a further access to the polarization by the so-called asymmetry method is possible, directly from the line shape. Plotting the determined polarization against the respective area units, as shown in Fig. 30, leads to a linear correlation, where the slope is the known enhancement factor $k$. Comparing the two enhancement factors $\left(k_{T E}=12.22 \pm 0.57\right.$ and $\left.k_{\text {asy }}=12.39 \pm 0.07\right)$ identified for this sample, shows no major contradictions and can thus be used for a confirmation of the correct operation of the module.

\subsection{Conclusion}

The various tests have shown that the newly developed cw NMR-module is ready for experimental use and even smallest signals can be well detected. First measurements were performed with a trityl-doped D-butanol target in Mainz and have shown no discrepancies. The high compatibility with the Liverpool module also ensures an easy deployment in existing test set-ups, therefore an additional alternative for the polarization detection is available.

Finally with the new NMR module we have build an experimental tool to measure the nucleon polarization with high presission for single or double polrization experiments. It will be the NMR 'standard' for upcoming polarized target set-ups and polarization experiments.

\section{Conclusions and Outlook}

Here we reported on latest developments of the collaborative work of the polarized target groups at the RuhrUniversität Bochum and the Universität Bonn within the CRC16 subproject D1 'Polarized solid state target'. Over the last 12 years both groups always pursued the objective to increase the 'figure of merit' and the luminosity of the polarized target for polarization experiments at various particle beams and experiments. While the Bochum group concentrated on dedicated target material research to maximize the nucleon polarization especially in deuterated samples, the Bonn group focused its research program on the development of the so called ' $4 \pi$ continuous 

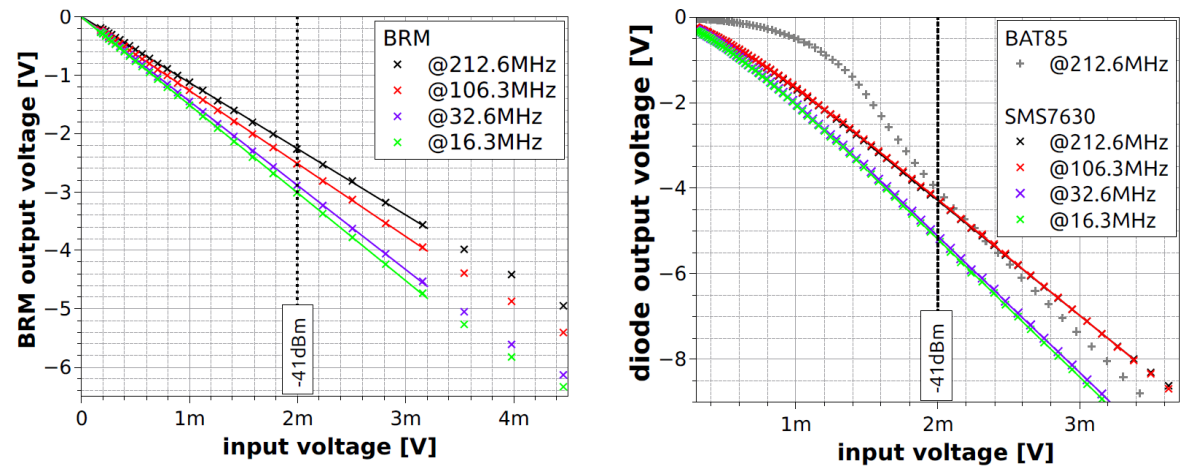

Figure 28: Measuring results for both channels for the observation of the linearity at selected frequencies. As can be seen, a constant gain of the diode channel based on BAT85 diodes was not given. However, by the exchange by SMS7630 diodes, the effect could be eliminated, so that both channels show a high degree of linearity in the measuring range at about $2 \mathrm{mV}$ input voltage ( $\sim-41 \mathrm{dBm}$ input power $)$.
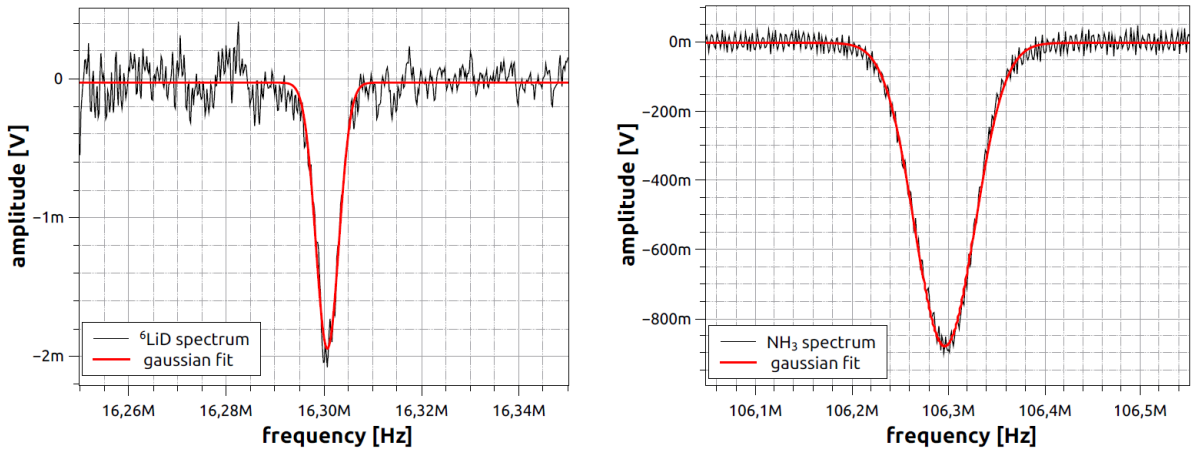

Figure 29: NMR spectra of deuterons ( $\left.{ }^{6} \mathrm{LiD} @ 77 \mathrm{~K}: P_{t}=0.0007 \%\right)$ and protons (ammonia @ $\left.1 \mathrm{~K}: P_{t}=0.255 \%\right)$ in thermal equilibrium at $2.5 \mathrm{~T}$. As can be seen, even smallest signals can be well detected, enabling a sufficiently accurate measurement for a TE-calibration.
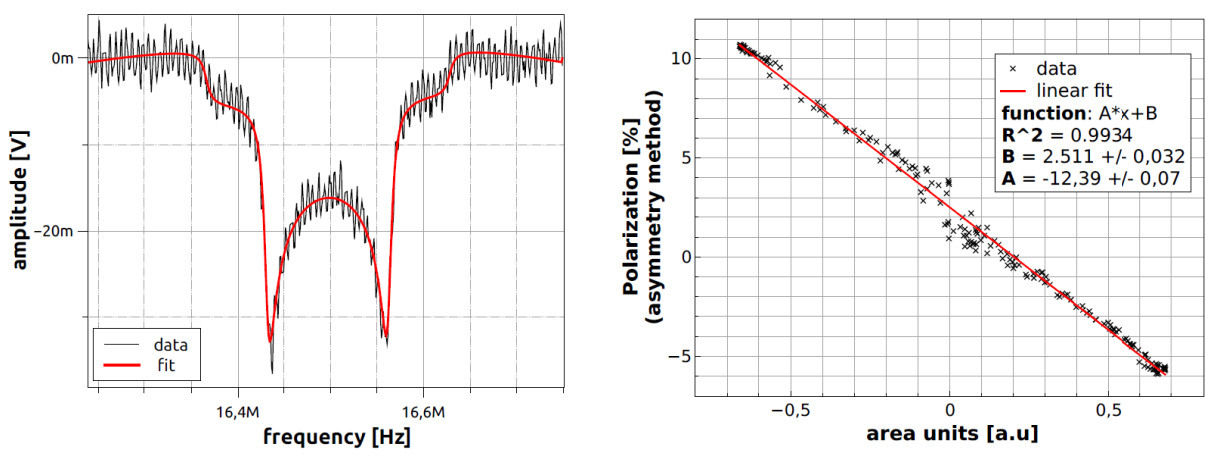

Figure 30: The TE spectra of quadrupole broadened D-butanol and the calculated polarization versus the determined area units of signals. As can be seen, there is an almost linear relationship, thereby providing a high linearity of this channel is illustrated. 
mode polarized target'. Nowadays the research activities led to highest proton and deuteron polarization values up to $P_{p} \sim 90 \%$ and $P_{d} \sim 85 \%$ in scattering experiments and a frozen spin target facility with large angular acceptance (close to $4 \pi$ ) which provides all possible polarization directions by means of the internal superconducting holding coils. This technique has been developed by the Bonn polarized target group in the early 1990s, long before the $\mathrm{CRC} 16$, and is nowadays the standard frozen spin spin technique at the accelerator facilities in Bonn, Mainz and JLAB. The ' $4 \pi$ continuous mode polarized target' extend the range of the frozen spin technique to higher beam intensities (by a factor of 1000) and data taking efficiency and will set the new standard for the application of the polarized solid state target in scattering experiments using $4 \pi$-detection systems.

The work reported here has been supported by the Deutsche Forschungsgemeinschaft within the SFB/TR16.

\section{References}

[1] S. Goertz, W. Meyer and G. Reicherz, Polarized H, $D$ and ${ }^{3}$ He targets for particle physics experiments, Prog. Part. Nucl. Phys. 49, 403-489 (2002)

[2] W. Hillert, The Bonn Electron Stretcher Accelerator ELSA: Past and future, EPJ A28, 139 (2006)

[3] W. Hillert, in this proceedings, (2016)

[4] M. Goldman, Spin Temperature and Nuclear Magnetic Resonance in Solids, Oxford University Press, (1970)

[5] G. Reicherz et al., Pulsed NMR for the determination of the nuclear polarization, NIM A526, 96 (2004)

[6] Ch. Hess, Ein gepulstes NMR-System zur Polarizationsmessung an Festkörpertargets, Diploma thesis, RUB (2005)

[7] St. Goertz et al., Highest polarizations in deuterated compounds, NIM A526, 43 (2004)

[8] St. Goertz, Spintemperatur und magnetische Resonanz verdünnter elektronischer Systeme - ein Weg zur Optimierung polarizierter FestkörperTargetmaterialien, Habilitation thesis, RUB (2002)

[9] J. Heckmann, Electron spin resonance and its implication on the maximum nuclear polarization of deuterated solid target materials, Phys. Rev. B74, 134418 (2006)

[10] J. Heckmann et al., Recent Progress in the Dynamic Nuclear Polarization of Solid Deuterated Butanol Targets, Appl. Magn. Reson. 34, 461 (2008)

[11] Ch. Bradtke et al., A new frozen-spin target for $4 \pi$ particle detection, NIM A436 430 (1999)

[12] H. Dutz et al., First measurement of the GerasimovDrell-Hearn sum rule for ${ }^{1} \mathrm{H}$ from 0.7 to $1.8 \mathrm{GeV}$ at ELSA, Phys. Rev. Lett. 91, 192001 (2003)

[13] Ch. Rohlof and H. Dutz, Effective densities and polarizations of the targets for the GDH-experiments at MAMI and ELSA, NIM A526, 126 (2004)
[14] H. Dutz, Summary of the $9^{\text {th }}$ International Workshop on polarized solid state targets and techniques, Trieste, Italy 2004, World Scientific 221 (2005)

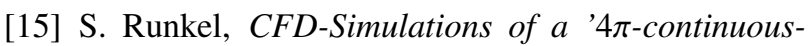
mode' dilution refrigerator for the CB-ELSA experiment, $\mathrm{XVI}^{\text {th }}$ International Workshop in Polarized Sources, Targets, and Polarimetry, PoS(PSTP2015)018, Bochum (2015)

[16] J. H. Ferziger, M. Peric, Computational Methods for Fluid Dynamics, Springer, Berlin (2002)

[17] H.K. Veersteg, W. Malalasekera, An introduction to Computational Fluid Dynamics, Longman Scientific \& Technical, Harlow (1995)

[18] C.Bradtke, A New Frozen-Spin Target for the Measurement of Helicity Asymmetry of the Total Photoabsorption Cross-Section, PHD-Thesis, Universität Bonn (2000)

[19] W. Polifke and J.Kopitz, Wärmeübertragung: Grundlagen, analytischer und numerische Methoden, Pearson Studium, 2nd edition, chapter 8, Munich, Germany (2009)

[20] H. Oertel, Prandtl - Führer durch die Ströhmungslehre, Springer, Wiesbaden (2012)

[21] G. Frosatti, Experimental Techniques: Methods for Cooling Below $300 \mathrm{mK}$, Journal of Low Temperature Physics Vol. 87, 3 (1992)

[22] Frank Pobell, Mater and Methods at Low Temperatures, Springer 3rd. edition, Berlin (2007)

[23] OpenFOAM Foundation, OpenFOAM User Guide, Version 2.3.0 (2014)

[24] J. P. Van Doormal and G. D. Raithby, Enhancement of the SIMPLE Method for Predicting compressible Flows, Num. Heat Trans 7, 147-163 (xxxx)

[25] NIST, Cryogenics Technologies Group, Website (2010), http://cryogenics.nist.gov/MPropsMAY/materialproperties.htm

[26] NIST , Chemical Web book, Website (2010), http://webbook.nist.gov/chemistry/fluid/

[27] W. E. Keller, ${ }^{3} \mathrm{He}$ and ${ }^{4} \mathrm{He}, 2$ ed., Plenum Press (1969).

[28] Ch. Rohlof, Entwicklung polarisierter Targets zur Messung der Gerasimov-Drell-Hearn-Summenregel an ELSA, PhD thesis, Universität Bonn (2003)

[29] B. Feher, Entwicklung eines supraleitenden, kryostatinternen Polarizationsmagneten für polarizierte Targets, Diploma thesis, Universität Bonn (2010)

[30] M. Bornstein, Bau und Test eines kryostatinternen supraleitenden Polarizationsmagneten, DiplomaThesis, Universität Bonn (2013).

[31] M. Bornstein, Development of a thin, internal superconducting polarisation magnet for the Polarised Target, $\mathrm{XVI}^{\text {th }}$ International Workshop in Polarized Sources, Targets, and Polarimetry, PoS(PSTP2015)006, Bochum (2015)

[32] Y. Iwasa, Case Studies in Superconducting Magnets, Second Edition, Springer US, New York (2009). 
[33] E. Kallenbach, et al. Elektromagnete -Grundlagen, Berechnung, Entwurf, 4. Edition, Vieweg + Teubner Verlag, Wiesbaden (2012).

[34] B. van den Brandt et al. Dynamic nuclear polarization in thin polymer foils and tubes, NIM A356, 36 (1995)

[35] B. van den Brandt et al., Dynamic nuclear polarization in thin polyethylene foils cooled via a superfluid ${ }^{4}$ He film, NIM A381, 219 (1996)

[36] E. I. Bunyatova, Free radicals and polarized targets, NIM A526, 22 (2004)

[37] Kumada et al., Dynamic nuclear polarization of high- and low-crystallinity polyethylenes, NIM A606, 669 (2009)

[38] S. Reeve, Polarisation and relaxation characteristics of irradiated polymeric materials at 1 $K$ and at $2.5 \mathrm{~T}, \mathrm{XVI}^{\text {th }}$ International Workshop in Polarized Sources, Targets, and Polarimetry, PoS(PSTP2015)017, Bochum (2015)

[39] D. Libby and M.G. Ormerod, Electron spin resonance spectrum of stretched polyethylene, Journal of Physics and Chemistry of Solids 18, 316 (1961)

[40] E.J. Lawton et al., Paramagnetic-Resonance Studies of Irradiated High-Density Polyethylene. I. Radical Species and the Effect of Environment on Their Behavior, The Journal of Chemical Physics 33, 395 (1960)

[41] H. Fischer and K.-H. Hellwege, Elektronenspinresonanz-Untersuchungen an bestrahltem Polypropylen, Journal of Polymer Science 56, 33 (1962)

[42] L. J. Forrestal and W. G. Hodgson, Electron spin resonance studies of irradiated polypropylene, Journal of Polymer Science Part A 2.3, 1275 (1964)

[43] M. Iwasaki et al., Electron spin resonance of trapped free radicals and the photoinduced radical conversion in $\gamma$-irradiated isotactic polypropylene, Journal of Polymer Science Part B: Polymer Letters 5.5, 423 (1967)
[44] S. Nara et al., Configurations of the free radicals in irradiated polypropylene and the relation of their decay reactions to the molecular motion, Journal of Polymer Science Part A-2: Polymer Physics 8.9, 929 (1970)

[45] S. Runkel, Herstellung und Untersuchung von Ammoniakkristallen als polariziertes Target für das COMPASS-Experiment, Univerität Bonn (2011)

[46] T. Fujimura et al., Conversion of alkyl radicals to allyl radicals in irradiated single crystal mats of polyethylene, Polymer 19, 1031 (1978)

[47] A. Meier, Entwicklung eines Kryo-Thermostaten hoher Kühlleistung zur Targetpräparation für das COMPASS-Experiment, Ruhr-Universität Bochum (1997)

[48] S. Reeve, A Wide Temperature Range Cryostat for Polarized Target Material Preparation, Univerität Bonn (2011)

[49] G. Reicherz et a., The Bonn polarized target NMRsystem, NIM A356, 74 (1995)

[50] J. Herick, Development of a new Q-meter module, $\mathrm{XVI}^{\text {th }}$ International Workshop in Polarized Sources, Targets, and Polarimetry, PoS(PSTP2015)011, Bochum (2015)

[51] F. Bloch, Nuclear Induction, Phys. Rev., 70, 460-474 (1946).

[52] R.K. Harris, Nuclear Magnetic Resonance Spectroscopy, Longman Scientific \& Technical, Harlow, 1986.

[53] G.R. Court et al., A high precision Q-meter for the measurement of proton polarization in polarized targets, NIM A324, 433-440 (1993).

[54] J. Herick, Ein neues cw-NMR-Modul und Trityldotierte D-Butanol-Targets für Streuexperimente, $\mathrm{PhD}$ thesis, Ruhr-Universität Bochum (2015).

[55] P. Hautle, Detection of small NMR signals, Proceedings of the Workshop on NMR in Polarized Targets, University of Virginia (1998).

[56] Ch.M. Dulya et al., A line-shape analysis for spin-1 NMR signals, NIM A398, 109-125 (1997). 